\title{
Recomendaciones para la atención odontológica de niños con discapacidad y riesgo médico durante la pandemia COVID-19
}

Asociación Latinoamericana de Odontopediatría. Equipo Interdisciplinario COVID-19

\section{Introducción}

En el marco de la actual situación mundial provocada por la pandemia de COVID-19 resulta imprescindible dedicar de forma prioritariala atención a niñosy adolescentes con discapacidad quienes se encuentran en una situación de vulnerabilidad al incluirse dentro de los grupos con mayor riesgo de complicaciones. ${ }^{1,2}$ Las medidas de protección frente a la pandemia que se adopten en este grupo no sólo estarán destinadas a los niños sino a sus familias y cuidadores.

El objetivo de este artículo es proporcionar recomendaciones y consideraciones para la atención odontológica de niños con discapacidad y con condiciones médicas de riesgo evaluando el impacto en la salud general y las implicancias emocionales y psicológicas que la pandemia tiene sobre ellos y sus familias y/o cuidadores.

\section{Consideraciones generales}

Según UNICEF (2020), ${ }^{1}$ en el contexto de la pandemia de COVID-19, los niños con discapacidad pueden tener un mayor riesgo de exposición y complicaciones.
Esto se basa en la consideración de diversos factores que incluyen: condiciones de salud subyacentes que aumentan el riesgo de complicaciones graves de COVID-19, y condiciones socioeconómicas, ya que la población de niños con discapacidad está representada desproporcionadamente entre las personas del mundo que viven en la pobreza, por lo que los impactos de COVID-19 pueden ser peores. Además pueden verse afectados desfavorablemente por la interrupción de los servicios de salud y apoyos domésticos, comunitarios y sociales, incluida la asistencia personal y lamentablemente se enfrentan a obstáculos para acceder a las medidas de prevención y respuesta a sus necesidades. ${ }^{3}$

Negrini S. y colaboradores, ${ }^{4}$ brindaron los primeros datos sobre el impacto de COVID-19 en las personas con discapacidad en Europa. Concluyen que la pandemia está teniendo una gran repercusión en los servicios de salud ya que los pacientes no reciben atención debido al cierre de servicios ambulatorios, por lo que sufren lo que llaman un "daño colateral". La rehabilitación de estos niños se centra en el funcionamiento y la calidad 
de vida y se debe aceptar una reducción de las actividades a favor de las acciones y necesidades para salvar vidas por un corto período de tiempo. De esto se deduce que si este período de falta de intervención de los equipos médicos y de apoyo se extiende, las consecuencias deteriorarán la calidad de vida de esta población. 5,6

La OMS ${ }^{7}$ describe las consideraciones sobre la discapacidad durante el confinamiento por COVID-19, enfatizandoen los riesgos de contagio por distintas causas que incluyen barreras parala implementacióndemedidas básicas de higiene, como el lavado de manos (ej. imposibilidad para una persona con discapacidad motora); dificultad para promulgar el distanciamiento social debido a discapacidad intelectual o porque son pacientes institucionalizados; por la necesidad de tocar cosas para obtener información del entorno (discapacidad visual o intelectual) o para soporte físico (discapacidad motora) enfatizando también en las barreras para acceder a la información de salud pública y de acceso a la atención médica. ${ }^{8}$

En momentos de estrés e incertidumbre, como los se enfrentan en esta pandemia, es importante tener estrategias para manejar distintas situaciones que pueden afrontar los niños con discapacidad, como por ejemplo los cambios abruptos en las rutinas, la pérdida de conexiones con entornos amigables (amigos, terapeutas), la incapacidad de aceptar el confinamiento, que pueden llevar a presentar sintomatología como ansiedad o depresión. ${ }^{9}$ Por ello tanto la familia y cuidadores, así como los profesionales de salud, entre ellos los odontopediatras, juegan un papel importante para ayudar a comprender los cambios y procesar sus sentimientos relacionados.

Por ejemplo, en casos de niños con TEA (Trastornos del Espectro Autista) Hume, K. y colaboradores ${ }^{10}$ proponen una estrategia de siete ítems para apoyar a las personas con autismo durante estos tiempos inciertos: 1. Apoyo a la comprensión, 2. Ofrecer oportunidades de expresión, 3. Dar prioridad a las habilidades de afrontamiento y relajación, 4. Mantener rutinas, 5. Construir nuevas rutinas, 6. Fomentar conexiones (desde la distancia), 7. Manejar los cambios de comportamiento. En muchas ciudades del mundo se han otorgado permisos especiales para pacientes con TEA $u$ otras discapacidades para realizar paseos acompañados con una persona a fin de evitar síntomas de ansiedad $\mathrm{u}$ otros. ${ }^{11}$

Es importante arbitrar los medios que permitan la continuidad de los controles médicos de niños con enfermedades sistémicas asociadas, como por ejemplo Síndrome de Down. ${ }^{12}$ Quienes en un 40 a $60 \%$ presentan cardiopatías congénitas y en casos severos, compromiso respiratorio. ${ }^{13}$

\section{Estrategia de atención odontopediátrica durante la etapa de confinamiento de la pandemia COVID-19.}

Esta estrategia se basa en las recomendaciones de ALOP - Asociación Latinoamericana de Odontología Pediátrica - referida a ruta de atención para procedimientos de Odontología Pediátrica durante la etapa de confinamiento o 
cuarentena de la pandemia COVID-19. ${ }^{14} \mathrm{~A}$ esta guía se añaden las recomendaciones pertinentes de acuerdo a las diferentes necesidades de los niños con discapacidad y riesgo médico asociado, siempre teniendo en cuenta que sólo se realizará atención odontológica de urgencias (Figura 1).

\section{Teleodontología}

La atención odontológica a distancia (teleodontología ${ }^{15}$ ), es la primera línea de la atención en casos excepcionales que se pueden presentar en una crisis de salud pública y cuando no es posible el contacto presencial entre el paciente y el especialista por distancias geográficas $u$ otras restricciones. ${ }^{15,16}$

La Teleodontología debe considerarse como una herramienta fundamental para poder evaluar el grado de urgencia/ emergencia para la atención odontológica. La utilización de esta modalidad en Odontopediatría en niños con condiciones

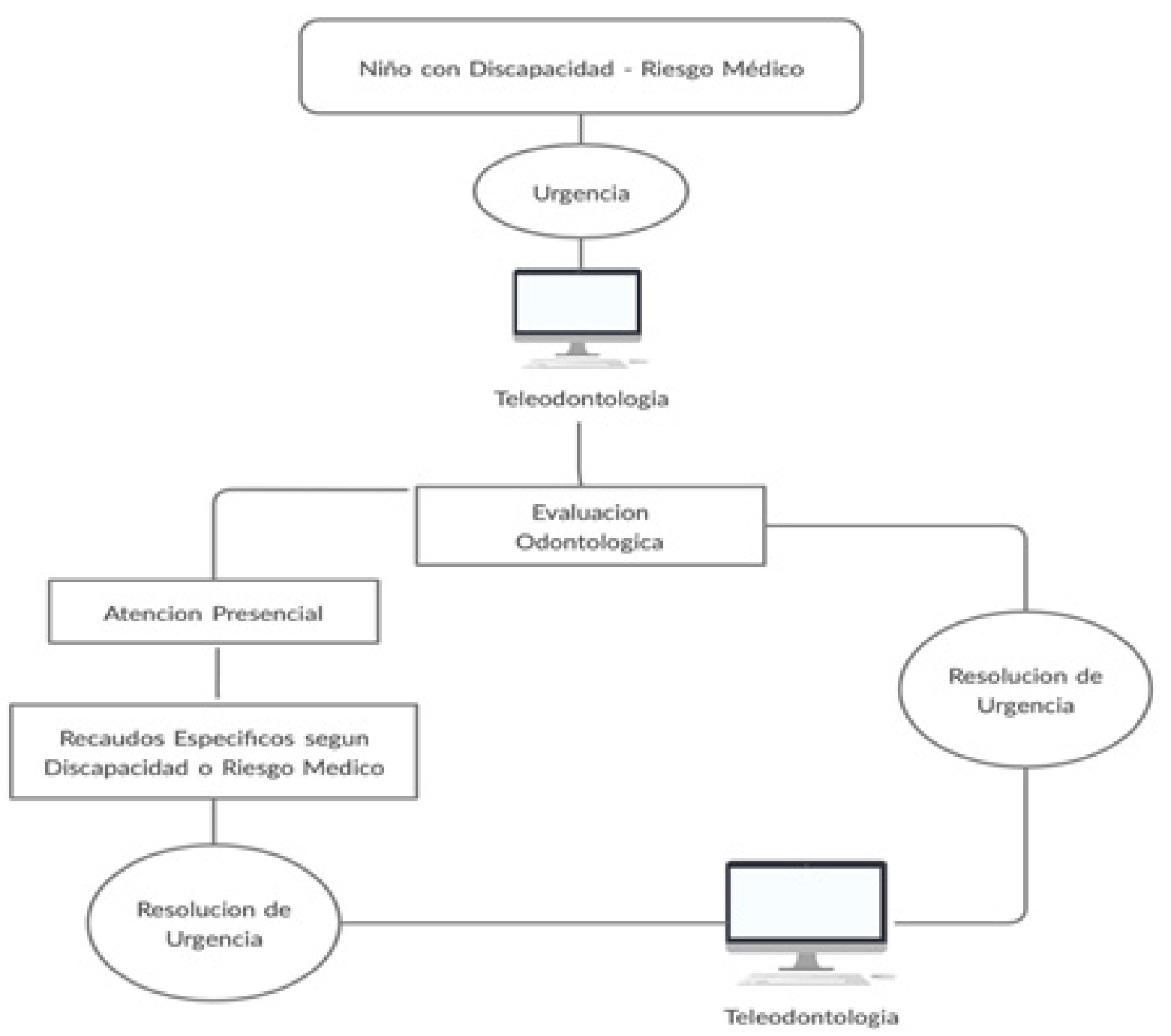

Figura 1. Flujograma de intervención para la resolución de urgencias odontológicas en el paciente con situación de discapacidad. 
de discapacidad o riesgo médico asociado seguirá los lineamientos de ALOP 2020,15 remarcando la importancia de poder resolver la urgencia por esta vía y continuar con el seguimiento virtual y de este modo evitar el riesgo de traslado debido a que constituyen un grupo de mayor riesgo en caso de contagio de SARS-CoV-2.

El interrogatorio estará destinado principalmente a los padres o cuidadores de los niños, $y$, en aquellos pacientes que presenten riesgo médico, se deberá realizar la interconsulta con su equipo de salud correspondiente. Es importante en estos casos solicitar el contacto del médico de cabecera a fin de realizar las interconsultas en caso de ser necesarias. Se realizará el triage virtual siguiendo la "Ficha de seguimiento de Urgencia odontopediátrica durante la pandemia COVID-19" (ALOP $2020^{15}$ ) y de esta manera se obtendrá información de la signosintomatología del paciente a fin de brindar orientación y decidir la vía de seguimiento.

La aplicación de la atención mediante telemedicina / teleodontología debe contemplar que existen brechas en el acceso a las computadoras y a Internet y limitaciones en las habilidades para su uso en los hogares con personas con discapacidad. ${ }^{17}$ En algunos niños puede ocurrir que sea difícil para el odontopediatra reconocer la urgencia específica por diferentes factores, por ejemplo, una conducta no colaboradora que impida obtener imágenes intrabucales aceptables, o por la imposibilidad de la familia o cuidadores de comprender o descifrar la causa de la urgencia. En estos casos será preciso organizar la atención en forma ambulatoria y presencial para la resolución de la urgencia. Una vez decidida la citación del paciente se intercambian ideas con los padres o cuidadores a fin de preparar al niño para la concurrencia. Para ello se puede enviar material (videos, pictogramas, etc.) de acuerdo a la condición de cada paciente a fin de poder reforzar la motivación que se realizará en forma presencial.

\section{Cita presencial}

En este trabajo se sugieren distintas estrategias para adaptar a los niños a la situación odontológica ${ }^{17}$ de urgencia, teniendo en cuenta las condiciones de discapacidad o enfermedad sistémica compleja que presenten, utilizando estrategias de modelado conductual y motivacional, y además teniendo en cuenta tanto la protección del personal de salud (odontólogos, asistentes y administrativos) como la del niño y su acompañante para evitar la transmisión del SARS-CoV-2. ${ }^{19,20}$

\section{Antes del ingreso al consultorio odontológico:}

El odontopediatra estará vestido con ambo (vestido quirúrgico) y usando mascarilla quirúrgica o inclusiva ${ }^{21}$ la cual permite la visualización de la boca y su movimiento al hablar, la cual es recomendada por ejemplo para niños hipoacúsicos.

Se respetarán siempre los dos metros de distancia al recibir al paciente en la puerta del consultorio. Se tomará la temperatura y hará el triage presencial con un interrogatorio que incluya la condición médica del niño. La asistente dental se encontrará vestida con el equipo de protección personal $\mathrm{EPP}^{22,23}$ para asistir al odontólogo a vestirse con las mismas 
medidas de bioseguridad (EPP). Se recomienda que el niño con discapacidad intelectual o trastorno del espectro autista (TEA) presencie, con la distancia física indicada, toda la maniobra de colocación de los diferentes elementos que debe usar el profesional (Protocolo indicado en Ruta de atención para procedimientos de Odontología Pediátrica $\mathrm{ALOP}^{14}$ ) y así disminuir el temor y la ansiedad que pueda ocasionar la vestimenta requerida para la atención. Se sugiere evitar excesos en la utilización de vestimenta que sólo agregan más posibilidades de equivocaciones al descartar la misma y no aportan realmente mayor bioseguridad.

Se recomienda trabajar con anterioridad los procedimientos antes explicados a través de la Teleodontología, entregando distintos recursos a los padres o cuidadores para lograr la adaptación al entorno odontológico. Se debe explicar la secuencia del procedimiento a los padres o cuidadores a fin de poder trabajar los pasos con el niño en su hogar. Debe considerarse la posible dificultad en la comunicación y en la accesibilidad cognitiva e implementar la comunicación aumentativa, mediante el uso de pictogramas para poder así explicar el origen de esta enfermedad, síntomas, hábitos de higiene y nuevas rutinas. ${ }^{24,25}$ En la cita presencial se utilizarán los mismos pictogramas o videos antes del ingreso al consultorio para reforzar la motivación o adaptación del niño a la nueva situación. $^{26,27}$ La institución ARASAAC pone a disposición en su página materiales para ofrecer a las familias, terapeutas y profesionales (Figuras 2 y 3 ). ${ }^{28}$

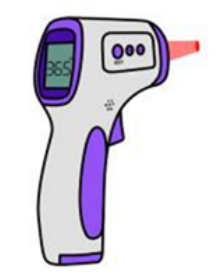

Termómetros

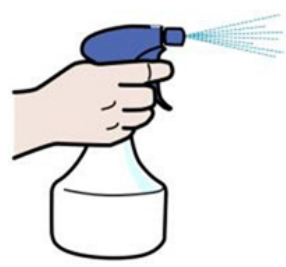

Sanitizante para superficies y ropa

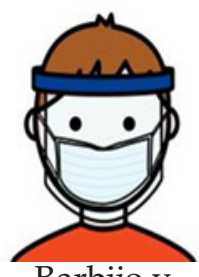

Barbijo y mascarilla.
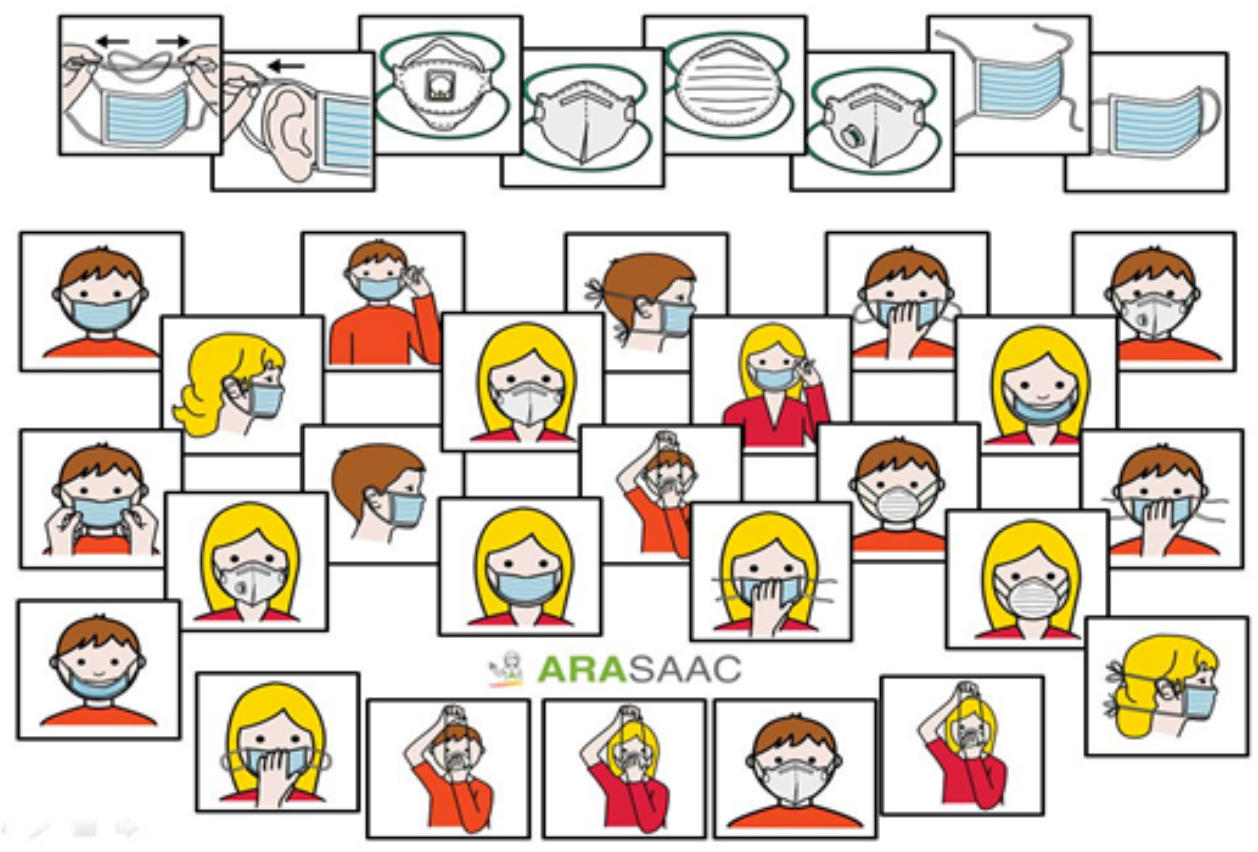

Figura 2. Pictogramas de materiales y mascarillas Fuente: ARASAAC http://www.arasaac.org/materiales.php?id_material=2357 


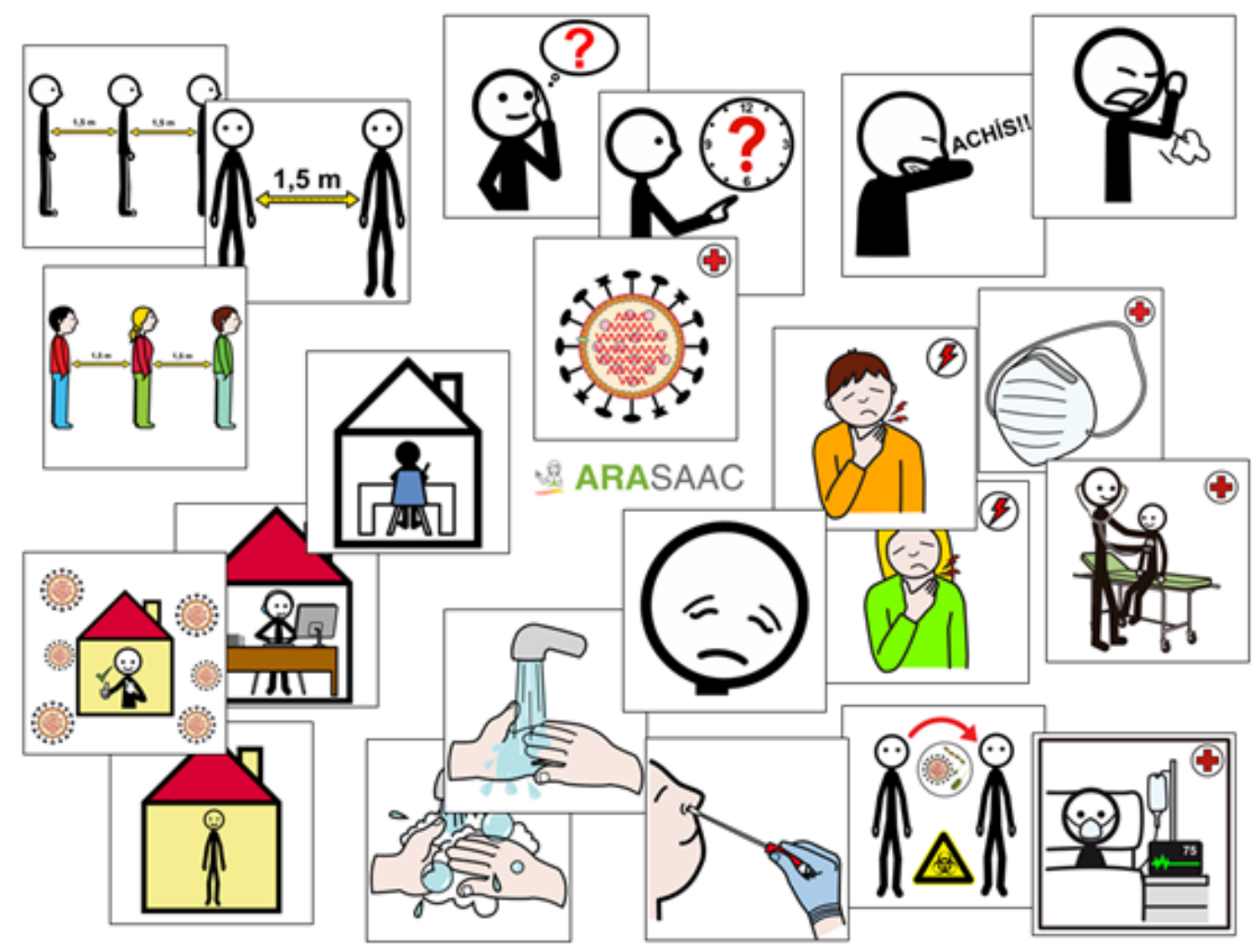

Figura 3. Pictogramas de conductas recomendadas durante la pandemia COVID-19.

Fuente: $h t t p: / / w w w . a r a s a a c . o r g / m a t e r i a l e s . p h p ? i d \_m a t e r i a l=2357$

\section{En el consultorio odontológico:}

1. Antes de comenzar la atención al paciente, este debe hacer un enjuague con colutorio de peróxido de hidrógeno al $1 \%$ o un colutorio de iodopovidona al $0.2 \%$ durante 1 minuto, (previo descarte de alergia al iodo). En la mayoría de los pacientes pediátricos menores de 4 años, así como en aquellos niños con discapacidad motora, intelectual, sensorial o con difícil conducta no se indica enjuagatorio por la incapacidad de realizarlo, o para disminuir el riesgo de ingestión; es por ello que se realiza la antisepsia de la cavidad bucal con gasa, rollo de algodón o cepillo impregnados en la solución empleada. La elección de la modalidad de higiene se puede decidir con los padres o cuidadores.
2. En casos de pacientes con ECNE (Encelopatía crónica no evolutiva) o con otras alteraciones motoras se sugiere, previo al procedimiento intraoral, realizar masajes orofaciales a fin de disminuir la espasticidad y relajar al niño en forma apropiada. ${ }^{29}$

3. El uso de instrumental rotatorio deberá minimizarse, priorizando procedimientos que no generen aerosoles. ${ }^{30,31}$ Se debe considerar que los niños con TEA o con trastornos sensoriales son muy sensibles a los ruidos y se prefiere el abordaje de caries de mínima invasión como por ejemplo con técnicas como la atraumática (TRA) o la aplicación de diamino fluoruro de plata. ${ }^{30,32}$

4. El uso del dique de goma para 
procedimientos operatorios o endodónticos, estará indicado siempre que sea posible. En algunos casos se puede utilizar cianoacrilato, bandas elásticas, hilo dental para reemplazar el uso de clamps o grapas. ${ }^{33,34}$

5. Utilizar alta succión si es posible. El ruido puede desencadenar conductas no deseadas al igual que el uso de instrumental rotatorio en niños con trastornos sensoriales y con gran sensibilidad por lo que se recomienda utilizar baja succión o auriculares además de música para disminuir la percepción. $^{35}$

6. Mantener el trabajo a 4 manos es indispensable. ${ }^{31}$ Una asistente dental entrenada es pilar fundamental tanto en la atención odontológica como en la ayuda durante la motivación del niño con discapacidad y contención de sus padres o cuidadores.

7. Durante el tratamiento sólo deberán encontrarse el paciente, operador $y$ asistente en el consultorio. De ser indispensable, permitir máximo un acompañante por paciente. En pacientes que lo permitan puede mantenerse la puerta abierta del consultorio y el acompañante estar en el umbral de la puerta para que el niño pueda observarlo y hasta participar de la situación odontológica calmando al paciente.

8. El paciente debe ser capaz de colaborar con el tratamiento que se realiza. Para ello utilizar las estrategias de adaptación del niño a la consulta. En casos de pacientes que no tengan habilidad para colaborar con el tratamiento, derivar para atención bajo sedación o anestesia general.$^{31,36,37}$ En los casos de derivación a esa modalidad de atención el niño no debe presentar patología respiratoria aguda (COVID-19 sintomático), y se controlará la urgencia odontológica con medidas sintomáticas (control de la infección y el dolor con antimicrobianos y analgésicos sí corresponde).

Se ha expresado preocupación con respecto al riesgo de la generación de aerosol en la administración de analgesia de óxido nitroso pero hasta el momento, no hay estudios directamente relacionados con este problema potencial. ${ }^{31,36}$ Las guías clínicas aconsejan el uso de óxido nitroso y otras formas de sedación consciente si se requiere atención urgente en pacientes que no permiten el tratamiento en forma convencional. A su vez se recomienda evitar utilización de anestesia general para sólo utilizarla en pacientes con pobre salud bucal que pueda impactar y agravar condiciones médicas pre-existentes así como en niños con comportamientos autoagresivos debido al dolor. ${ }^{31,38}$

\section{Conclusión}

La odontopediatría debe asegurar accesibilidad a la atención odontológica a los niños con discapacidades o enfermedades sistémicas de riesgo conociendo el impacto emocional, psicológico y físico que esta pandemia de COVID 19 está provocando en la población en general y en estos niños en particular.

Por esta razón, resulta necesario que el odontopediatra anticipe junto a las familias y el equipo interdisciplinario las nuevas 
rutinas para facilitar la atención tomando medidas como la Teleodontología previa a la consulta, preparando a los niños para aceptar el entorno odontológico con las medidas de bioseguridad apropiadas así como propiciando el uso de comunicación alternativa alusiva a la actual pandemia y de esta manera contribuir a la desensibilización del niño a la hora de afrontar la atención odontológica.

\section{Autores}

Desarrollo de este artículo liderado por Gabriela Scagnet (Argentina), Jorgelina Valente (Argentina), Ana Clara Zabala (Argentina), Mariana Armada (Argentina), Laura Fedelli (Argentina), Lina María Hernández Salas (Colombia), como parte del Equipo Interdisciplinario COVID-19 de la Asociación Latinoamericana de Odontopediatría.

Equipo Interdisciplinario COVID-19 de la Asociación Latinoamericana de Odontopediatría:

Jenny Abanto (Brasil), Mariana Armada (Argentina), Paola Beltri (España), Marisol Carrillo Tabakman (Paraguay), Haydée Casaretto (Argentina), Jorge Luis Castillo (Perú), Mónica Gladys Cesetti (Argentina), Bertha Angélica Chávez González (Perú), Ana Claudia Rodrigues Chibinski (Brasil), Salomon Alberto Cohen (Argentina), Olga Cortés
Lillo (España), Luzia Ana Da Silva de Carballo (Venezuela), Gonzalo De la Fuente Alvarez (Chile), Renée Di Nallo (Argentina), María Débora Elizabeth Dricas (Argentina), Sandra Echevarria (Brasil), Piedad Cecilia Echeverry Marin (Colombia), Laura Fedelli (Argentina), Carlos Flores-MIr (Canadá), Andrea Virgina González Carfora (Chile), Lina María Hernández Salas (Colombia), Francisco José Hernández Restrepo (Colombia), Maria Teresa Ibañez Rodriguez (Bolivia), José Carlos Pettorossi Imparato (Brasil), Alejandra Lipari Valdés (Chile), Daniela Madrigal López (Costa Rica), Daniela Catalina Martínez Camus (Chile), María Gabriela Martínez Vásquez (Venezuela), A. Carolina Medina Díaz (Venezuela), Kelly Maria Silva Moreira (Brasil), Elías M. Morón (Estados Unidos de América), Martha Mutis (Estados Unidos de América), Camila Palma (Perú), Gladys Mabel Peña (Argentina), Adriana Pistochini (Argentina), Paloma Planells (España), Gabriel Politano (Brasil), Matias RiosErazo (Chile), Adriana Maria Rubiano Pinzon (Colombia), Karla Mayra Rezende (Brasil), Rosa Gabriela Rondón (Venezuela), Gabriela Scagnet (Argentina), Rosemary Sogbe de Agell (España), Marina Tavares Costa Nóbrega (Canadá), Jorgelina Valente (Argentina), Ernesto Venegas De Herrera (República Dominicana), Rosa Helena Wanderley Lacerda (Brasil), Ana Clara Zabala (Argentina), Gabriel Zambrano (Venezuela).

Información completa del Equipo Interdisciplinario COVID-19 de la Asociación Latinoamericana de Odontopediatría

\section{Referencias bibliográficas}

1. United Nations International Children's Emergency Fund. UNICEF. Respuesta COVID-19: consideraciones para niñas, niños y adultos con discapacidades.2020. Disponible en :https://www. unicef.org/disabilities/files/RESPUESTA_COVID_-_SPANISH.pdf

2. Morales-Chávez M. Coronavirus y discapacidad - Una población muy vulnerable. Acta Odontol. Venez. Edición Especial: COVID-19. 2020.

3. Campbell VA, Gilyard JA, Sinclair L, Sternberg T, Kailes JI. Preparing for and responding to pandemic influenza: Implications for people with disabilities. Am. J. Public Health. 2009; 99(S2): S294-S300. Disponible en: https://ajph.aphapublications.org/doi/10.2105/AJPH.2009.162677

4. Negrini S, Grabljevec K, Boldrini P, Kiekens C, Moslavac S, Zampolini M, et al. Up to 2.2 million people experiencing disability suffer collateral damage each day of Covid-19 lockdown in Europe. Eur J Phys Rehabil Med. 2020; May 08. DOI: 10.23736/S1973-9087.20.06361-3. Disponible en: https://doi. org/10.23736/S1973-9087.20.06361-3

5. European Physical and Rehabilitation Medicine Bodies Alliance, Negrini S. White Book on Physical and Rehabilitation Medicine in Europe. Introductions, Executive Summary, and Methodology. European J Phys Rehabil Med. 2018; 54(2): 125-155. Disponible en: https://doi.org/10.23736/S1973-9087.18.05143-2. 
6. World Health Organization.WHO. Critical preparedness, readiness and response actions for COVID-19. Disponible en: https://www.who.int/publications-detail/critical-preparedness-readinessand-response-actions-for-covid-19

7. World Health Organization. WHO: Disability considerations during the COVID-19 outbreak Disponible en: : https://www.paho.org/en/documents/disability-considerations-during-covid-19-outbreak

8. Noticias Organización Naciones Unidas. ONU. Las personas con discapacidad, en riesgo por el coronavirus. [Internet] [Consultado 19 Marzo 2020]. Disponible en: https://news.un.org/es/ story/2020/03/1471282

9. Hume K, Waters V, Sam A, Steinbrenner J, Perkins Y, Dees B., et al. Supporting individuals with autism through uncertain times. Chapel Hill, NC: School of Education and Frank Porter Graham Child Development Institute, University of North Carolina at Chapel Hill. 2020. Disponible en :A https:// afirm.fpg.unc.edu/supporting-individuals-autism-through-uncertain-times

10. Lima CKT, Carvalho PMM, Lima IAAS, Nunes JVAO, Saraiva JS, de Souza RI, et al. The emotional impact of Coronavirus 2019-nCoV (new Coronavirus disease). Psychiatry Res. 2020; 12:112915.

11. Autism Spaeks.USA: Autism Speak; what should the autism community know about the coronavirus outbreak?; c2020; [cited 2020 March 4] Disponible en : https://www.autismspeaks.org/science-news/ what-should-autism-community-know-about-coronavirus-outbreak

12. Scagnet G. Actualización odontológica en la atención del niño con síndrome de Down. Odontol. pediatr. 2013; 12(1): 27-40. Spanish. Disponible en: http://repebis.upch.edu.pe/articulos/op/v12n1/a4.pdf

13. Medrano CL., García-Guereta LS, Lirio JC, García JP. Respiratory infections, Down's syndrome and congenital heart disease: the CIVIC 21 study. An Pediatr. 2009; 71(1): 38-46. Español. Disponible en : https://doi.org/10.1016/j.anpedi.2008.11.007

14. Asociación Latinoamericana de Odontopediatría. Grupo COVID-19.Ruta de atención para procedimientos de Odontología Pediátrica durante la etapa de confinamiento o cuarentena de la pandemia COVID-19. 2020; 10 (2).Disponible en: https://www.revistaodontopediatria.org

15. Asociación Latinoamericana de Odontopediatría. Grupo COVID-19. Tele dentistry Guidelines in Pediatric Dentistry during the COVID-19 pandemic. 2020; 10, (2).Disponible en: https://www. revistaodontopediatria.org/ediciones/2020/2/art-3

16. Jampani ND, Nutalapati R, Dontula BS, Boyapati R. Applications of teledentistry: A literature review and update. J Int Soc Prev Community Dent. 2011; 1(2):37-44. doi:10.4103/2231-0762.97695.

17. Economic Commission for Latin America and the Caribbean. ECLAC. Persons with disabilities and coronavirus disease (COVID-19) in Latin America and the Caribbean: status and guidelines.2020. Disponible en: https://www.cepal.org/en/publications/45492-persons-disabilities-and-coronavirusdisease-covid-19-latin-america-and-caribbean

18. Polli V, Sordi M, Lisboa M, Munhoz E, Camargo A. Dental Management of Special Needs Patients: A Literature Review . Glob J Oral Sci.2016 ; (2):33-45.

19. Centers for Disease Control and Prevention. CDC.Coronavirus Disease 2019; Interim infection prevention and control recommendations for patients with suspected or confirmed coronavirus disease 2019 (COVID-19) in healthcare settings. 2020; March 19. Disponible en: https://www.simlaweb. it/wp-content/uploads/2020/03/Infection-Control-Severe-acute-respiratory-syndrome-coronavirus-2SARS-CoV-2-CDC.pdf

20. American Academy of Pediatric Dentistry. COVID-19 Update/Coronavirus Update. 2020; April. Disponible en : https://www.aapd.org/about/about-aapd/news-room/covid-19/.

21. Federación de Asociaciones de Personas con Discapacidad Física y Orgánica de Córdoba. COCEMFE. Mascarillas solidarias. 2020; April. Disponible en: https://cordobainclusiva.com/mascarilla-solidaria

22. Centers for Disease Control and Prevention CDC. Recommendation: Postpone Non-Urgent Dental Procedures, Surgeries, and Visits. 2020; March 27. Disponible en: https:/www.cdc.gov/oralhealth/ infectioncontrol/statement-COVID.html.

23. Verbeek JH, Rajamaki B, Ijaz S, Sauni R, Toomey E, Blackwood B, Tikka C, Ruotsalainen JH, Kilinc Balci FS.; Cochrane Collaboration; Cochrane Database of Systematic Reviews. Personal protective equipment for preventing highly infectious diseases due to exposure to contaminated body fluids in healthcare staff. 2020(5) CD011621. Disponible en: https://www.cochranelibrary.com/es/cdsr/ doi/10.1002/14651858.CD011621.pub3/full/es 
24. Confederación Autismo España. Gobierno de Aragón: Centro Aragonés para la Comunicación Aumentativa y Alternativa. ARASAAC. 2020. Disponible en : http://www.autismo.org.es/actualidad/ articulo/como-explicar-el-covid-19-las-personas-con-tea:\%20ARASAAC (www.arasaac.org)

25. Gobierno de Aragón: Centro Aragonés para la Comunicación Aumentativa y Alternativa. ARASAAC. Pictogramas de conductas recomendadas durante la pandemia COVID-19. (http:// arasaac.org Licencia: CC (BY-NC-SA) Disponible en: https://drive.google.com/file/d/1uLDQMF_ rGn3VPxkWmqGJYFVv89EWkuOD/view

26. Aspanaes. Material accesible para personas con TEA para comprender el Coronavirus. Pictograma. 2020. Disponible en: https://drive.google.com/file/d/1MC1HuPq3KHywm4z7rKDgB-rcjYnzUOdE/ view.

27. Molina M. COVIBOOK .2020. Available from: https://660919d3-b85b-43c3-a3ad-3de6a9d37099. filesusr.com/ugd/64c685_0a595408de2e4bfcbf1539dcf6ba4b89.pdf open?id=10xY03EV8mvK78m09NR5Pm2qB1uJ0xDgq:

/https://drive.google.com/

28. Centro Aragonés para la Comunicación Aumentativa y Alternativa ARASAAC. Disponible en: http:// aulaabierta.arasaac.org/materiales-caa-coronavirus)

29. García, M. D. E., \& Zambrano, V. P. P. Parálisis cerebral infantil: estimulación temprana del lenguaje método de Bobath. Dominio de las Ciencias. 2017. Jun 20; 3(4), 627-706.

30. AsociaciónLatinoamericanadeOdontopediatría:TratamientodecariesenépocadeCOVID-19:Protocolos clínicos para el control de generación de aerosoles. Revista de Odontopediatría Latinoamericana. 2020; (10) 2. Disponible en: https://www.revistaodontopediatria.org/ediciones/2020/2/art-2/

31. International Association for Disability \& Oral Health. IADH. Second Edition of the IADH Fact Sheet COVID-19. 2020; April 15. Disponible en: http://iadh.org/news/updated-iadh-covid-fact-sheet-2-1/

32. Mallineni SK, Innes NP, Raggio DP, Araujo MP, Robertson MD, Jayaraman J. Coronavirus disease (COVID-19): Characteristics in children and considerations for dentists providing their care. Int . Paediatr Dent. 2020; 30(3), 245-250.

33. Torres Diaz, E. (2017). Aislamiento del campo operatorio en odontopediatría http://repositorio.uigv. edu.pe/handle/20.500.11818/1572.

34. González González JM. Cianoacrilato: Definición y propiedades. Toxicidad y efectos secundarios. Aplicaciones en medicina y odontología. Av Odontoestomatol. 2012; 28(2): 95-102.

35. Guzman G, Putrino N, Martinez F, Quiroz N. Nuevas tecnologías: Puentes de comunicación en el trastorno del espectro autista (TEA). Ter Psicol.2017; 35(3): 247-258. Disponible en: https://scielo. conicyt.cl/pdf/terpsicol/v35n3/0716-6184-terpsicol-35-03-0247.pdf . Spanish

36. Souza, R. C. C., Costa, P. S., \& Costa, L. R. Precauções e recomendações sobre sedação odontológica durante a pandemia de COVID-19. Rev Bras Odontol. April 2020; 77. Portuguese

37. Giordano CE, Giordano CL, Cunha-Correia AS. Sedação inalatória com óxido nitroso para assistência odontológica durante a pandemia de COVID-19. Revista Faipe. 2020; 10(1): 69-84. Disponible en: http://revistafaipe.lifesistemas.com.br/index.php/RFAIPE/article/view/196/141 Portuguese

38. Royal College of Surgeons of England, Recommendations for Special Care Dentistry during Covid-19 pandemic. April 2020. Disponible en : www.rcseng.ac.uk/dental-faculties/fds/coronavirus/

Recibido/Received: 01/06/2020

Modificaciones aceptadas/Final version: 07/06/2020

Correspondencia: e-mail: editor@revistaodontopediatria.org 


\section{Recomendações ao atendimento odontológico em crianças com necessidades especiais e alterações sistêmicas durante a pandemia de COVID-19}

\section{Introdução}

No contexto da atual situação mundial causada pela pandemia de COVID-19 é fundamental dedicar atenção prioritária a crianças e adolescentes com necessidades Especiais e em situação vulnerável, incluindo grupos com maior risco de complicações. Medidas de proteção em face da pandemia adotadas neste grupo, não visarão apenas crianças, mas também a suas famílias e cuidadores.

O objetivo deste artigo é fornecer recomendações e considerações para atendimento odontológico a crianças com necessidades especiais e alterações sistêmicas e avaliar o impacto na saúde geral e implicações emocionais e psicológicas que a pandemia tem sobre eles e suas famílias e / ou cuidadores.

\section{Considerações gerais}

De acordo com a UNICEF (2020), ${ }^{1}$ durante a pandemia da COVID-19, crianças com deficiências/Necessidades Especiais podem ter um risco aumentado de contágio e complicações. ${ }^{1,2}$ Isto é embasado considerando diversos fatores que incluem as condições de saúde destas crianças que aumentam risco de graves complicações com a COVID-19 e também condições sócio-econômicas podem influenciar.
Considerando também que a população de crianças com deficiência está representada de forma desproporcional entre as pessoas que vivem na pobreza em todo mundo, os impactos do COVID-19 podem ser piores neste grupo. Podem também serem afetados devido à interrupção dos serviços de saúde e assistência social, cuidados pessoais e infelizmente, enfrentam obstáculos para acessar medidas de prevenção e resposta a acesso a suas necessidades. ${ }^{3}$

Negrini S. e cols., ${ }^{4}$ contribuíram para fornecer os primeiros dados sobre impacto do COVID-19 nas pessoas com deficiência na Europa. Concluem que a pandemia de COVID-19 está tendo um grande impacto nos serviços de saúde, uma vez que os pacientes não recebem atendimento, devido ao fechamento de serviços ambulatoriais, e com isso sofrem o que podemos chamar de "dano colateral". A reabilitação dessas crianças se concentra na recuperação da função e qualidade de vida e deve aceitar uma redução de atividades em favor de ações e necessidades para salvar vidas, mas por um curto período de tempo, mas se esse período se prolongar, sem o suporte necessário das equipes médicas e de assistência, as consequências desta população de crianças com necessidades especiais, sofrerão uma deterioração da qualidade de vida. ${ }^{5,6}$ 
A $\mathrm{OMS}^{7}$ descreve considerações sobre incapacidade durante o confinamento COVID-19, enfatizando os riscos de contágio de diferentes causas, incluindo dificuldades de implementação de medidas básicas de higiene, como lavar roupas, mãos (por exemplo, Por serem pessoas com deficiência motora); Essas situações impedem o distanciamento social necessário, devido a deficiência intelectual ou porque são pacientes institucionalizados; alguns precisam tocar em objetos nos ambientes pois possuem (deficiência visual ou intelectual) ou para apoio físico (deficiência motora) também enfatizando as barreiras ao acesso à informação em saúde pública e acesso a cuidados médicos. ${ }^{8}$

Em tempos de estresse e incertezas, como os que enfrentamos nesta pandemia, é importante ter estratégias para lidar com as diferentes situações que crianças com deficiência podem enfrentar, como as mudanças bruscas nas rotinas, perda de conexões com ambientes amigáveis (amigos, terapeutas), incapacidade de aceitar e entender oconfinamento pode desencadear sintomas como ansiedade ou depressão. ${ }^{9}$ Portanto, familiares e cuidadores, bem como profissionais da saúde, incluindo odontopediatras, desempenham um papel importante na compreensão dessas mudanças e processar seus sentimentos relacionados a esta situação.

Por exemplo, em crianças com TEA (Transtornos do Espectro do Autismo) Hume, K. e cols, ${ }^{10}$ propõem estratégias para esses casos em sete itens de apoios a pessoas com autismo durante estes tempos de incertezas: 1. Apoio à compreensão, 2. Oferecer oportunidades de expressão. 3. Dê prioridade a enfrentamento e relaxamento, 4. Manutenção de rotinas, 5. Construção de novas rotinas.
6. Promover conexões (à distância) 7. Gerenciar mudanças de comportamento.

Autorizações especiais foram emitidas pelas autoridades em muitas cidades ao redor do mundo, durante o isolamento provocado pela pandemia, para que pessoas com TEA ou outras deficiências pudessem fazer caminhadas, acompanhadas com uma pessoa para reduzir ansiedade ou outros sintomas. ${ }^{11}$

É importante arbitrar os meios para que os controles médicos das crianças com doenças sistêmicas associadas, como por exemplo a síndrome de Down ${ }^{12}$ que em 40 a $60 \%$ dos casos têm cardiopatias congênitas e em casos graves, comprometimento respiratório. ${ }^{13}$

\section{Estratégia de atendimento odontológico pediátrico no confinamento da pandemia de COVID-19}

Essa estratégia é baseada nas recomendações da ALOP - Associação Latino-Americana de Odontopediatria - encaminhada para atendimento de procedimentos de Odontopediatria durante a fase de confinamento ou quarentena na Pandemia de COVID-19. ${ }^{14}$ Neste gráfico adicionaremos as recomendações que consideramos pertinentes de acordo com as diferentes necessidades das crianças com deficiência e alterações sistêmicas associadas, tendo sempre em mente que apenas realizaremos atendimento odontológico de urgências e emergências. (Figura 1)

\section{Tele-Odontologia}

O atendimento odontológico a distância (teleodontologia ${ }^{15}$ ) é a primeira linha de atenção em casos excepcionais que possam 


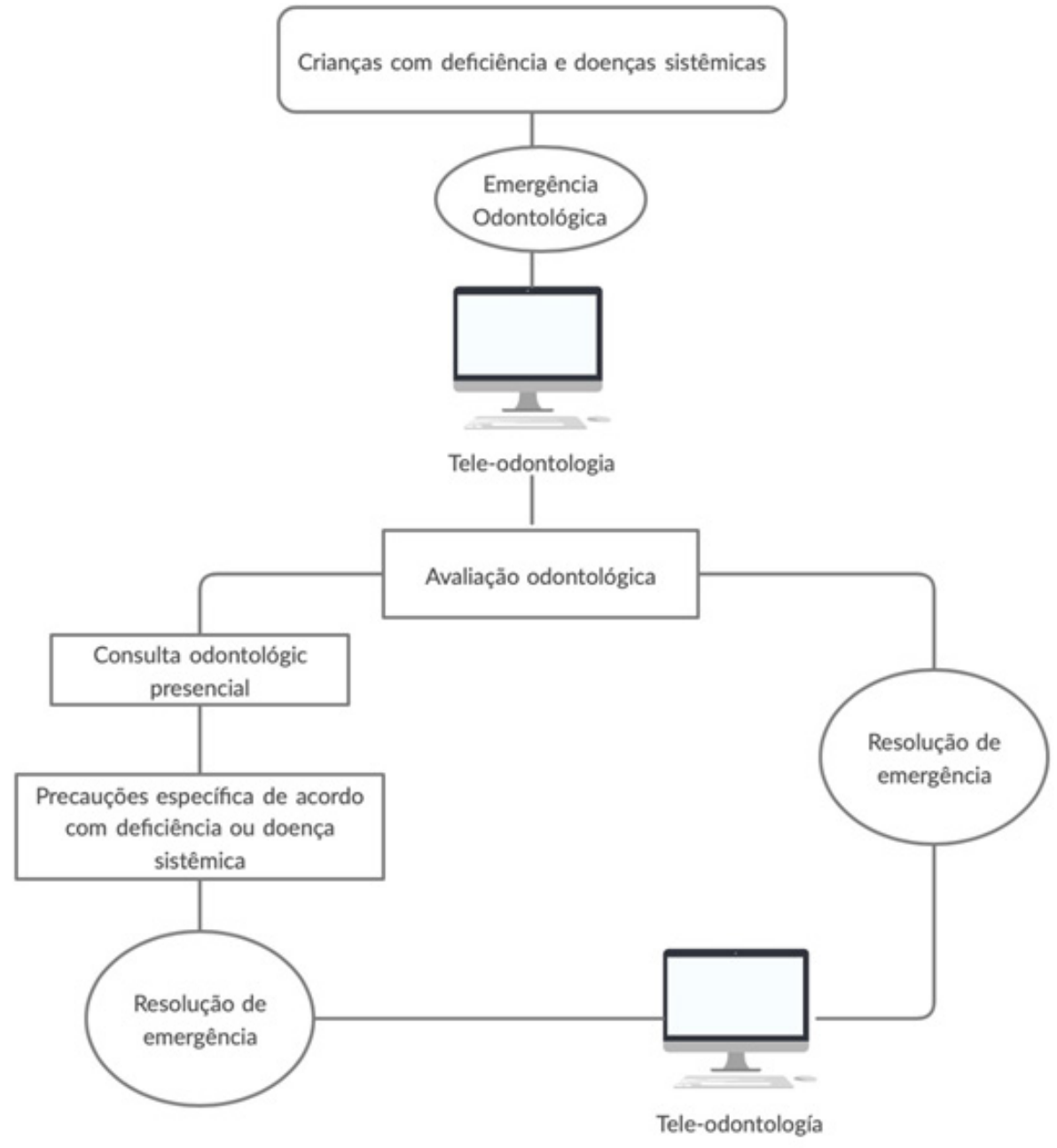

Figura 1. Fluxograma de intervenção para resolução de emergências odontológicas nos pacientes com deficiência.

surgir em crises de saúde pública e quando o contato frente a frente entre o paciente e o especialista não for possível devido a distâncias geográficas ou outras restrições. ${ }^{15,16}$

A tele-odontologia deve ser considerada como uma ferramenta fundamental para poder avaliar o grau de urgência / emergência para atendimento odontológico.

A Utilização desta modalidade em Odontopediatria em crianças com condições de incapacidade ou alterações sistêmicas associadas seguirá as diretrizes da ALOP 2020, ${ }^{15}$ destacando a importância de resolver a emergência e continuar com o acompanhamento virtual e, assim, evitar o risco de transferência devido à constituição de um grupo de risco aumentado em caso de infecção por SARS-CoV-2.

A entrevista será direcionada principalmente aos pais ou responsáveis das crianças e naqueles pacientes que apresentam alterações sistêmicas deverá ser feita interconsulta com a equipe médica correspondente 
É importante nesses casos solicitar o contato do médico da família, a fim de realizar as interconsultas em caso de necessidade.

A triagem virtual será realizada seguindo o "formulário de acompanhamento de Emergência pediátrica "durante a pandemia de COVID-19" (ALOP 202015) e desta maneira, obtemos informações sobre a sintomatologia do paciente, a fim de fornecer orientação e decidir o caminho do acompanhamento dos próximos passos.

A aplicação do cuidado por telemedicina / teleodontologia deve contemplar que existem lacunas no acesso a computadores e à Internet e limitações de habilidades para uso em residências com pessoas com deficiência. ${ }^{17} \mathrm{Em}$ algumas crianças, pode ser difícil para odontopediatra reconhecer a urgência específica para diferentes fatores, por exemplo, comportamento não colaborativo que impede a obtenção de imagens intraorais aceitáveis ou devido à impossibilidade da família ou cuidadores entendem ou decifram a causa da emergência. Nestes casos, será necessário organizar o atendimento ambulatorial e resolver a urgência pessoalmente.

Uma vez decidido o atendimento do paciente, as ideias são trocadas com os pais ou responsáveis, a fim de preparar a criança para a assistência odontológica. Para isso pode-se enviar material (vídeos, pictogramas, etc.) de acordo com a condição de cada paciente a fim de reforçar a motivação e o que será realizado na consulta ambulatorial.

\section{Nomeação Em Pessoa}

Neste trabalho, diferentes estratégias são sugeridas para adaptar as crianças à situação emergência odontológica, ${ }^{17}$ levando em consideração as condições de incapacidade ou doença sistêmica complexa que apresentam, usando estratégiasdemodelagem comportamental e motivacional, e também levando em consideração a proteção do profissional da saúde (dentistas, assistentes e pessoal administrativo), com a criança e seus acompanhantes para evitar a transmissão do SARS-CoV-2. ${ }^{19,20}$

\section{Antes de entrar no consultório odontológico:}

O odontopediatra vestirá ambos (avental cirúrgico), usará uma "face shield" (Viseira) que e máscara que permite a visualização da boca e seu movimento recomendado por exemplo para crianças com deficiência auditiva. ${ }^{21}$

Os dois metros de distância serão sempre respeitados ao receber o paciente à porta do consultório, a temperatura será medida e a triagem será feita com uma anamnese, incluindo a condição médica da criança. O assistente dental usará equipamento de proteção individual EPis $^{22,23}$ para participar do atendimento odontológico e auxiliará o dentista a vestir com as mesmas medidas de biossegurança (EPis), onde recomendamos que a criança com deficiência intelectual ou transtorno do espectro do autismo (TEA) com a distância física indicada, visualize toda a manobra de colocação dos diferentes elementos (EPis) que o profissional irá usar (Protocolo indicado no cuidados com os procedimentos de Odontopediatria $\mathrm{ALOP}^{14}$ ) e, assim, diminuir o medo e ansiedade que podem ser causados pelas roupas necessárias ao atendimento.

Sugere-se evitar o uso excessivo de roupas que apenas aumentará as chances de erro ao descartá-lo e realmente não fornecem um melhor biossegurança. 
Recomenda-se trabalhar os procedimentos explicados anteriormente através da Teleodontologia, fornecendo diferentes recursos aos pais ou cuidadores para alcançar adaptação ao ambiente odontológico. A sequência do procedimento para que os pais ou responsáveis possam trabalhar as etapas com as crianças em casa, podem encontrar alguma dificuldade de comunicação e acessibilidade cognitiva e podem implementar comunicação aumentativa, usando de pictogramas para explicar a origem desta doença, sintomas, hábitos de higiene e novas rotinas. ${ }^{24,25}$ No atendimento presencial, será usado os mesmos pictogramas ou vídeos antes de entrar no consultório para reforçar a motivação ou adaptação da criança à nova situação. ${ }^{26,27}$ A instituição ARASAAC coloca disponível em seu site, materiais para oferecer às famílias, terapeutas e profissionais (Figuras 2 e 3 ). ${ }^{28}$

\section{No consultório odontológico:}

1. Antes de iniciar $\mathrm{o}$ atendimento, $\mathrm{o}$ paciente deverá fazer bochecho com peróxido de hidrogênio a $1 \%$ ou enxaguatório bucal com iodopovidona $0,2 \%$ por 1 minuto (após descartar alergia ao iodo). $\mathrm{Na}$ maioria das crianças com menos de 4 anos de idade e crianças com deficiência motora, intelectual, sensorial ou nos pacientes com dificuldade conduta, nenhuma lavagem é indicada pela incapacidade de fazê-lo ou para diminuir o risco de ingestão, e por isso faz-se limpeza da cavidade oral com gaze, rolo de algodão ou pincel impregnado em peróxido hidrogênio. A escolha da modalidade de higiene pode ser decidida com os pais ou responsáveis.

2. Nos casos de pacientes com ECNE (encefalopatia crônica não evolutiva) ou com outras alterações motoras,

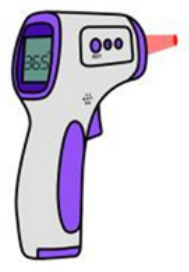

Termômetro
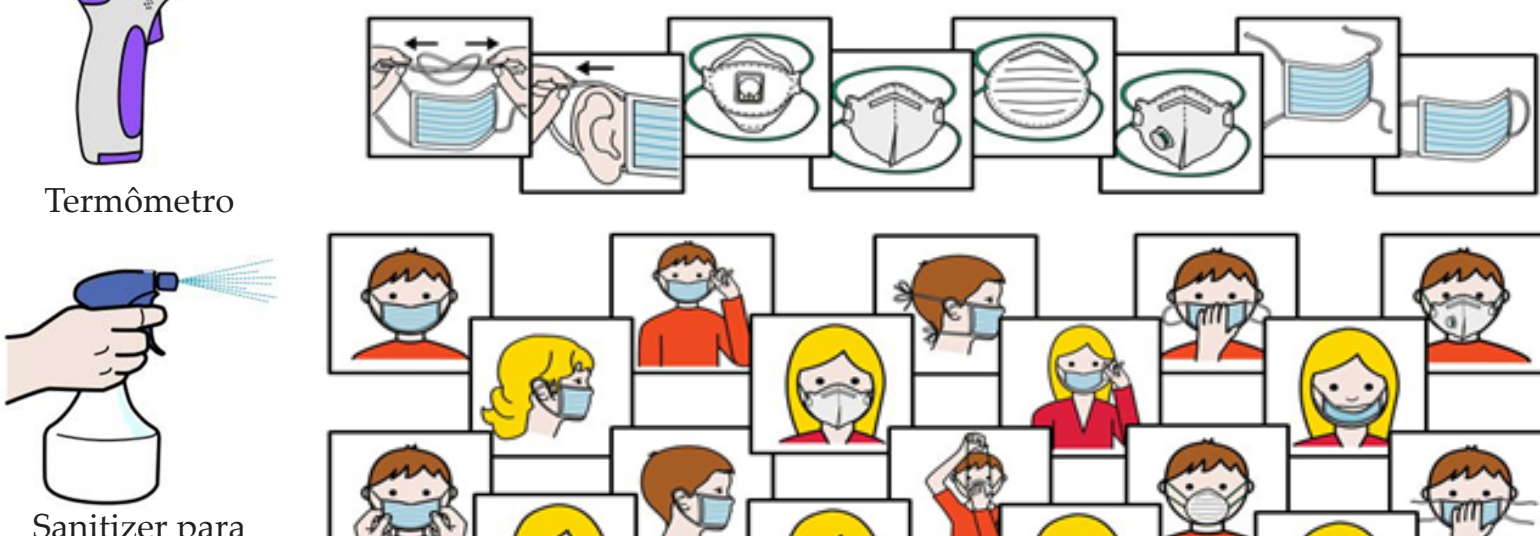

Sanitizer para superfícies
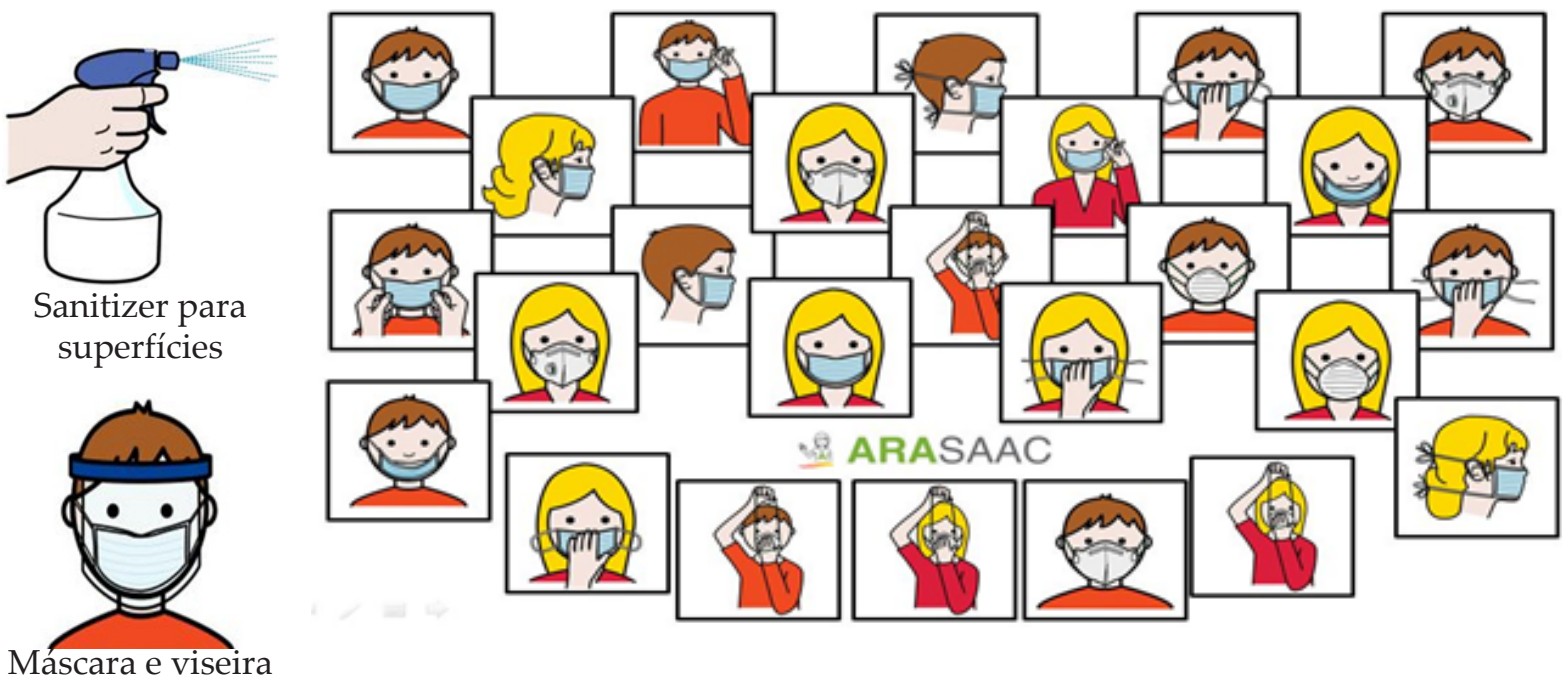

Figura 2. Pictogramas de materiais e máscaras. Fonte: ARASAAC http://www.arasaac.org/materiales.php?id_material=2357 


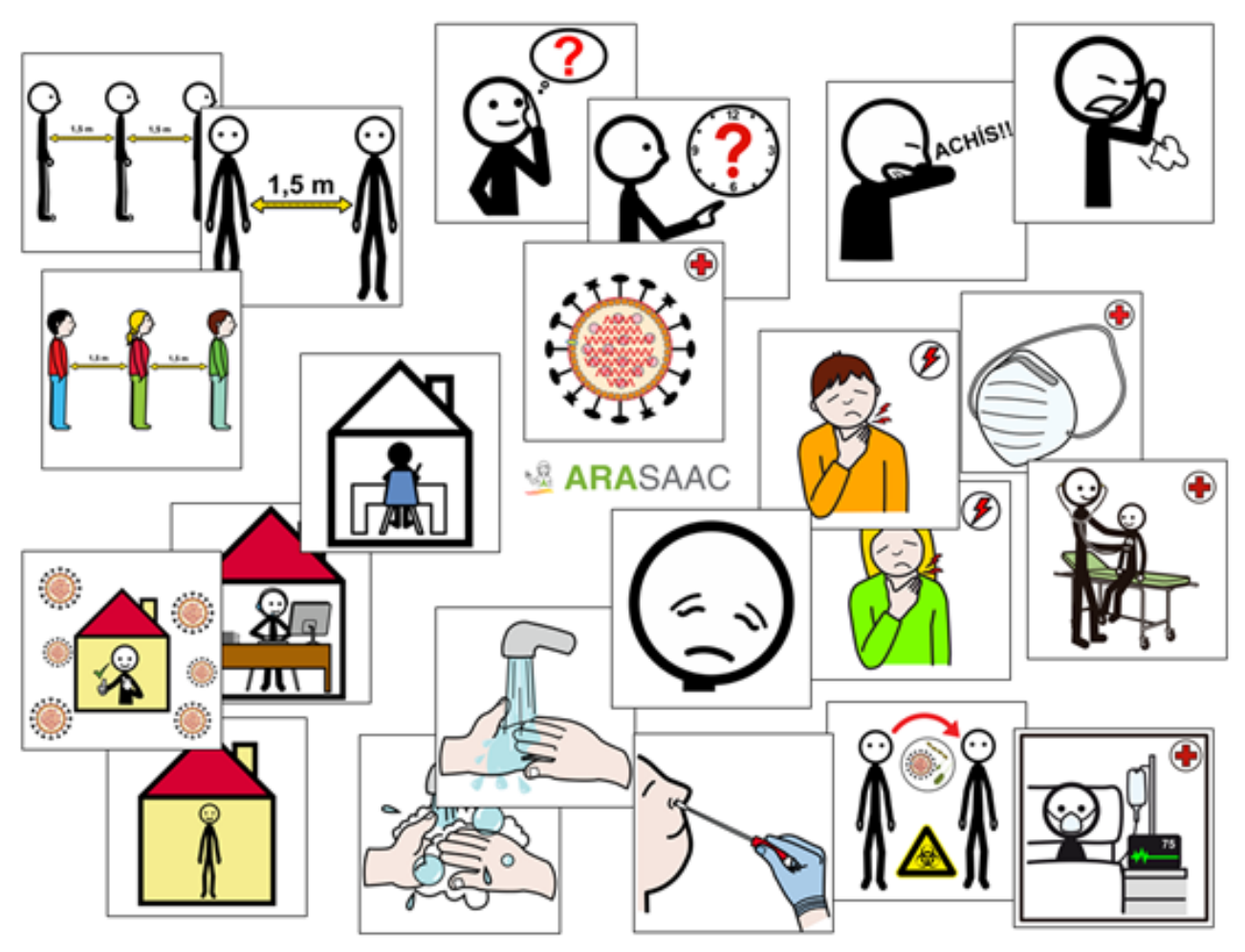

Figura 3. Pictogramas de comportamentos recomendados durante a pandemia do COVID-19. Fonte: ARASAAC http://www.arasaac.org/materiales.php?id_material=2357

sugere-se, antes do procedimento intraoral, realizar massagens orofaciais para reduzir a espasticidade e relaxar a criança de forma apropriada. ${ }^{29}$

3. O uso de instrumentos rotatórios deve ser minimizado, priorizando procedimentos que não geram aerossóis. ${ }^{30,31}$ Crianças com TEA ou com distúrbios sensoriais são muito sensíveis ao ruído e a abordagem de cárie minimamente invasiva, como a técnicas com ART atraumática ou a aplicação de fluoreto de diamina e prata $^{30,32}$ são recomendáveis.

4. O uso de isolamento absoluto com lençol de borracha para procedimentos operatórios ou endodônticos, será indicado sempre que possível. Em alguns casos, você pode usar cianoacrilato, elásticos, fio dental para substituir o uso de grampos ou grampos..$^{33,34}$
5. Use sugadores de alta sucção, se possível. O ruído pode desencadear comportamentos não desejado, bem como o uso de instrumentos rotatórios em crianças com distúrbios Sensoriais e altamente sensíveis, por isso é recomendado o uso de baixa sucção ou fones de ouvido e música para diminuir a percepção. ${ }^{35}$

6. Manter o trabalho a 4 mãos é essencial. $^{31} \mathrm{Um}(\mathrm{a})$ assistente dental treinado(a) é um pilar fundamental no atendimento odontológico e ajuda durante a motivação da criança deficiente e a contenção de seus pais e / ou cuidadores.

7. Durante o tratamento, deverá permanecer na sala de atendimento apenas o paciente, operador e assistente odontológica. Senecessário, permita no máximo 1 acompanhante, mantendose a porta aberta do consultório, o 
acompanhante poderá ser observado pela criança e até participar acalmando o paciente.

8. O paciente deve ser capaz de colaborar com o tratamento que está sendo realizado. Para isto utilizar estratégias de condicionamento da criança para a consulta. Nos casos de pacientes que não têm capacidade de colaborar com o tratamento, considere cuidados sob

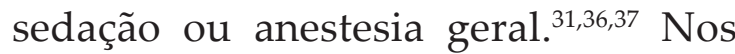
casos de encaminhamento para esse tipo de atendimento, a criança não deve apresentar patologia respiratório agudo (COVID-19 - sintomático) a urgência dentária será controlada com base nos sintomas (controle de infecção e/ou dor com uso de antimicrobianos e analgésicos).

Manifestou-se preocupação quantoao risco de geração de aerossóis na administração de analgesia por óxido nitroso, mas ainda não há estudos diretamente relacionado a esse possível problema. ${ }^{31}$ As diretrizes clínicas aconselham o uso de óxido nitroso e outras formas de sedação consciente, se necessário no atendimento de urgência em pacientes que não permitem tratamento convencional. Por sua vez, recomenda-se evitar o uso de anestesia geral para usálo apenas em pacientes com problemas de saúde bucal que podem impactar e agravar condições médicas pré-existente, bem como em crianças com comportamentos auto agressivos devido a dor. ${ }^{31,38}$

\section{Conclusão}

A Odontopediatria deve garantir a acessibilidade ao atendimento odontológico para crianças com deficiências ou doenças sistêmicas de risco, sabendo o impacto emocional, psicológico e físico que esta pandemia de COVID 19 está causando na população em geral e essas crianças em particular.

Por esse motivo, é necessário que o odontopediatra antecipe junto com as famílias e equipe interdisciplinar novas rotinas para facilitar o cuidado nas medidas como teleodontología antes da consulta, preparando as crianças para aceitar o ambiente odontológico com medidas adequadas de biossegurança, bem como promover o uso de comunicação alternativa, aludindo à pandemia atual e desta maneira de contribuir para a dessensibilização da criança diante do cuidado dental.

\section{Autores}

Esse artigo foi desenvolvido pelo grupo liderado: Gabriela Scagnet (Argentina), Jorgelina Valente (Argentina), Ana Clara Zabala (Argentina), Mariana Armada (Argentina), Laura Fedelli (Argentina), Lina María Hernández Salas (Colombia), como parte da Equipe Interdisciplinar COVID-19 da Associação Latinoamericana de Odontopediatria.

Equipe Interdisciplinar COVID-19 da Associação Latinoamericana de Odontopediatria:

Jenny Abanto (Brasil), Mariana Armada (Argentina), Paola Beltri (España), Marisol Carrillo Tabakman (Paraguay), Haydée Casaretto (Argentina), Jorge Luis Castillo (Perú), Mónica Gladys Cesetti (Argentina), Bertha Angélica Chávez González (Perú), Ana Claudia Rodrigues Chibinski (Brasil), Salomon Alberto Cohen (Argentina), Olga Cortés Lillo (España), Luzia Ana Da Silva de Carballo (Venezuela), Gonzalo De la Fuente Alvarez (Chile), Renée Di Nallo (Argentina), María Débora Elizabeth Dricas (Argentina), Sandra Echevarria (Brasil), Piedad Cecilia Echeverry Marin (Colombia), Laura Fedelli (Argentina), Carlos Flores-MIr (Canadá), Andrea Virgina González Carfora (Chile), Lina María Hernández Salas (Colombia), Francisco José Hernández Restrepo (Colombia), Maria Teresa Ibañez Rodriguez (Bolivia), José Carlos Pettorossi Imparato (Brasil), Alejandra Lipari Valdés (Chile), 
Daniela Madrigal López (Costa Rica), Daniela Catalina Martínez Camus (Chile), María Gabriela Martínez Vásquez (Venezuela), A. Carolina Medina Díaz (Venezuela), Kelly Maria Silva Moreira (Brasil), Elías M. Morón (Estados Unidos de América), Martha Mutis (Estados Unidos de América), Camila Palma (Perú), Gladys Mabel Peña (Argentina), Adriana Pistochini (Argentina), Paloma Planells (España), Gabriel Politano (Brasil), Matias Rios-Erazo (Chile), Adriana Maria Rubiano Pinzon (Colombia), Karla
Mayra Rezende (Brasil), Rosa Gabriela Rondón (Venezuela), Gabriela Scagnet (Argentina), Rosemary Sogbe de Agell (España), Marina Tavares Costa Nóbrega (Canadá), Jorgelina Valente (Argentina), Ernesto Venegas De Herrera (República Dominicana), Rosa Helena Wanderley Lacerda (Brasil), Ana Clara Zabala (Argentina), Gabriel Zambrano (Venezuela).

Información completa del Grupo Interdisciplinario COVID-19 de la Asociación Latinoamericana de Odontopediatría

\section{Referências}

1. United Nations International Children's Emergency Fund. UNICEF. Respuesta COVID-19: consideraciones para niñas, niños y adultos con discapacidades.2020. Disponible en :https://www. unicef.org/disabilities/files/RESPUESTA_COVID___SPANISH.pdf

2. Morales-Chávez M. Coronavirus y discapacidad - Una población muy vulnerable. Acta Odontol. Venez. Edición Especial: COVID-19. 2020.

3. Campbell VA, Gilyard JA, Sinclair L, Sternberg T, Kailes JI. Preparing for and responding to pandemic influenza: Implications for people with disabilities. Am. J. Public Health. 2009; 99(S2): S294-S300. Disponible en: https://ajph.aphapublications.org/doi/10.2105/AJPH.2009.162677

4. Negrini S, Grabljevec K, Boldrini P, Kiekens C, Moslavac S, Zampolini M, et al. Up to 2.2 million people experiencing disability suffer collateral damage each day of Covid-19 lockdown in Europe. Eur J Phys Rehabil Med. 2020; May 08. DOI: 10.23736/S1973-9087.20.06361-3. Disponible en: https://doi. org/10.23736/S1973-9087.20.06361-3

5. European Physical and Rehabilitation Medicine Bodies Alliance, Negrini S. White Book on Physical and Rehabilitation Medicine in Europe. Introductions, Executive Summary, and Methodology. European J Phys Rehabil Med. 2018; 54(2): 125-155. Disponible en: https://doi.org/10.23736/S1973-9087.18.05143-2.

6. World Health Organization.WHO. Critical preparedness, readiness and response actions for COVID-19. Disponible en: https://www.who.int/publications-detail/critical-preparedness-readiness-and-responseactions-for-covid-19

7. World Health Organization. WHO: Disability considerations during the COVID-19 outbreak Disponible en: : https://www.paho.org/en/documents/disability-considerations-during-covid-19-outbreak

8. Noticias Organización Naciones Unidas. ONU. Las personas con discapacidad, en riesgo por el coronavirus. [Internet] [Consultado 19 Marzo 2020]. Disponible en: https://news.un.org/es/ story/2020/03/1471282

9. Hume K, Waters V, Sam A, Steinbrenner J, Perkins Y, Dees B., et al. Supporting individuals with autism through uncertain times. Chapel Hill, NC: School of Education and Frank Porter Graham Child Development Institute, University of North Carolina at Chapel Hill. 2020. Disponible en :A https:// afirm.fpg.unc.edu/supporting-individuals-autism-through-uncertain-times

10. Lima CKT, Carvalho PMM, Lima IAAS, Nunes JVAO, Saraiva JS, de Souza RI, et al. The emotional impact of Coronavirus 2019-nCoV (new Coronavirus disease). Psychiatry Res. 2020; 12:112915.

11. Autism Spaeks.USA: Autism Speak; what should the autism community know about the coronavirus outbreak?; c2020; [cited 2020 March 4] Disponible en : https:/www.autismspeaks.org/science-news/ what-should-autism-community-know-about-coronavirus-outbreak

12. Scagnet G. Actualización odontológica en la atención del niño con síndrome de Down. Odontol. pediatr. 2013; 12(1): 27-40. Spanish. Disponible en: http://repebis.upch.edu.pe/articulos/op/v12n1/a4.pdf

13. Medrano CL., García-Guereta LS, Lirio JC, García JP. Respiratory infections, Down's syndrome and congenital heart disease: the CIVIC 21 study. An Pediatr. 2009; 71(1): 38-46. Español. Disponible en : https://doi.org/10.1016/j.anpedi.2008.11.007 
14. Asociación Latinoamericana de Odontopediatría. Grupo COVID-19.Ruta de atención para procedimientos de Odontología Pediátrica durante la etapa de confinamiento o cuarentena de la pandemia COVID-19. 2020; 10 (2).Disponible en: https://www.revistaodontopediatria.org

15. Asociación Latinoamericana de Odontopediatría. Grupo COVID-19. Tele dentistry Guidelines in Pediatric Dentistry during the COVID-19 pandemic. 2020; 10, (2).Disponible en: https://www. revistaodontopediatria.org/ediciones/2020/2/art-3

16. Jampani ND, Nutalapati R, Dontula BS, Boyapati R. Applications of teledentistry: A literature review and update. J Int Soc Prev Community Dent. 2011; 1(2):37-44. doi:10.4103/2231-0762.97695.

17. Economic Commission for Latin America and the Caribbean. ECLAC. Persons with disabilities and coronavirus disease (COVID-19) in Latin America and the Caribbean: status and guidelines.2020. Disponible en: https://www.cepal.org/en/publications/45492-persons-disabilities-and-coronavirusdisease-covid-19-latin-america-and-caribbean

18. Polli V, Sordi M, Lisboa M, Munhoz E, Camargo A. Dental Management of Special Needs Patients: A Literature Review . Glob J Oral Sci.2016;( 2):33-45.

19. Centers for Disease Control and Prevention. CDC.Coronavirus Disease 2019; Interim infection prevention and control recommendations for patients with suspected or confirmed coronavirus disease 2019 (COVID-19) in healthcare settings. 2020; March 19. Disponible en: https://www.simlaweb. it/wp-content/uploads/2020/03/Infection-Control-Severe-acute-respiratory-syndrome-coronavirus-2SARS-CoV-2-CDC.pdf

20. American Academy of Pediatric Dentistry. COVID-19 Update/Coronavirus Update. 2020; April. Disponible en : https://www.aapd.org/about/about-aapd/news-room/covid-19/.

21. Federación de Asociaciones de Personas con Discapacidad Física y Orgánica de Córdoba. COCEMFE. Mascarillas solidarias. 2020; April. Disponible en: https://cordobainclusiva.com/mascarilla-solidaria

22. Centers for Disease Control and Prevention CDC. Recommendation: Postpone Non-Urgent Dental Procedures, Surgeries, and Visits. 2020; March 27. Disponible en: https://www.cdc.gov/oralhealth/ infectioncontrol/statement-COVID.html.

23. Verbeek JH, Rajamaki B, Ijaz S, Sauni R, Toomey E, Blackwood B, Tikka C, Ruotsalainen JH, Kilinc Balci FS.; Cochrane Collaboration; Cochrane Database of Systematic Reviews. Personal protective equipment for preventing highly infectious diseases due to exposure to contaminated body fluids in healthcare staff. 2020(5) CD011621. Disponible en: https://www.cochranelibrary.com/es/cdsr/ doi/10.1002/14651858.CD011621.pub3/full/es

24. Confederación Autismo España. Gobierno de Aragón: Centro Aragonés para la Comunicación Aumentativa y Alternativa. ARASAAC. 2020. Disponible en : http://www.autismo.org.es/actualidad/ articulo/como-explicar-el-covid-19-las-personas-con-tea:\%20ARASAAC (www.arasaac.org)

25. Gobierno de Aragón: Centro Aragonés para la Comunicación Aumentativa y Alternativa. ARASAAC. Pictogramas de conductas recomendadas durante la pandemia COVID-19. (http:// arasaac.org Licencia: CC (BY-NC-SA) Disponible en: https://drive.google.com/file/d/1uLDQMF_ rGn3VPxkWmqGJYFVv89EWkuOD/view

26. Aspanaes. Material accesible para personas con TEA para comprender el Coronavirus. Pictograma. 2020. Disponible en: https://drive.google.com/file/d/1MC1HuPq3KHywm4z7rKDgB-rcjYnzUOdE/ view.

27. Molina M. COVIBOOK .2020. Available from: https://660919d3-b85b-43c3-a3ad-3de6a9d37099. filesusr.com/ugd/64c685_0a595408de2e4bfcbf1539dcf6ba4b89.pdf open?id=10xY03EV8mvK78m09NR5Pm2qB1uJ0xDgq:

28. Centro Aragonés para la Comunicación Aumentativa y Alternativa ARASAAC. Disponible en: http:// aulaabierta.arasaac.org/materiales-caa-coronavirus)

29. García, M. D. E., \& Zambrano, V. P. P. Parálisis cerebral infantil: estimulación temprana del lenguaje método de Bobath. Dominio de las Ciencias. 2017. Jun 20; 3(4), 627-706.

30. AsociaciónLatinoamericanadeOdontopediatría:TratamientodecariesenépocadeCOVID-19:Protocolos clínicos para el control de generación de aerosoles. Revista de Odontopediatría Latinoamericana. 2020; (10) 2. Disponible en: https://www.revistaodontopediatria.org/ediciones/2020/2/art-2/ 
31. International Association for Disability \& Oral Health. IADH. Second Edition of the IADH Fact Sheet COVID-19. 2020; April 15. Disponible en: http://iadh.org/news/updated-iadh-covid-fact-sheet-2-1/

32. Mallineni SK, Innes NP, Raggio DP, Araujo MP, Robertson MD, Jayaraman J. Coronavirus disease (COVID-19): Characteristics in children and considerations for dentists providing their care. Int . Paediatr Dent. 2020; 30(3), 245-250.

33. Torres Diaz, E. (2017). Aislamiento del campo operatorio en odontopediatría http://repositorio.uigv. edu.pe/handle/20.500.11818/1572.

34. González González JM. Cianoacrilato: Definición y propiedades. Toxicidad y efectos secundarios. Aplicaciones en medicina y odontología. Av Odontoestomatol. 2012; 28(2): 95-102.

35. Guzman G, Putrino N, Martinez F, Quiroz N. Nuevas tecnologías: Puentes de comunicación en el trastorno del espectro autista (TEA). Ter Psicol.2017; 35(3): 247-258. Disponible en: https://scielo. conicyt.cl/pdf/terpsicol/v35n3/0716-6184-terpsicol-35-03-0247.pdf . Spanish

36. Souza, R. C. C., Costa, P. S., \& Costa, L. R. Precauções e recomendações sobre sedação odontológica durante a pandemia de COVID-19. Rev Bras Odontol. April 2020; 77. Portuguese

37. Giordano CE, Giordano CL, Cunha-Correia AS. Sedação inalatória com óxido nitroso para assistência odontológica durante a pandemia de COVID-19. Revista Faipe. 2020; 10(1): 69-84. Disponible en: http://revistafaipe.lifesistemas.com.br/index.php/RFAIPE/article/view/196/141 Portuguese

38. Royal College of Surgeons of England, Recommendations for Special Care Dentistry during Covid-19 pandemic. April 2020. Disponible en : www.rcseng.ac.uk/dental-faculties/fds/coronavirus/

Recibido/Received: 01/06/2020

Modificaciones aceptadas/Final version: 07/06/2020

Correspondencia: e-mail: editor@revistaodontopediatria.org 


\section{Recommendations for dental care of children with disabilities and medically compromised during the COVID-19 pandemic}

\section{Introduction}

In the context of the current global situation caused by the COVID-19 pandemic it is essential to devote priority attention to children and adolescents with disabilities who are in a vulnerable situation, as they are included in the groups with the highest risk of complications. ${ }^{1,2}$ The pandemic protection measures adopted in this group will not only be aimed at children but also to their families and caregivers.

The purpose of this article is to provide recommendations and considerations for dental care of children with disabilities and medically compromised, assessing the impact on overall health and the emotional and psychological implications that the pandemic has on them and their families or caregivers.

\section{General considerations}

According to UNICEF (2020), ${ }^{1}$ in the context of the COVID-19 pandemic, children with disabilities may be at increased risk of exposure and complications. This is based on the consideration of various factors including: underlying health conditions that increase their risk of serious complications of COVID-19, and socioeconomic conditions as the population of children with disabilities is disproportionately represented among the world's people living in poverty 2 so the impacts of COVID-19 may be worse. They may also be adversely affected by the interruption of health services and domestic, community and social support, including personal assistance and unfortunately face obstacles in accessing prevention and response measures to their needs. ${ }^{3}$

Negrini S. et $a l^{4}$ provide the first data on the impact of COVID-19 on people with disabilities in Europe. They conclude that the pandemic is having a major impact on health services since patients do not receive care due to the closure of outpatient services, ${ }^{2}$ therefore they suffer what they call collateral damage. Rehabilitation therapies for these children are focused on functionality and improving quality of life. Reduction of these activities in favor of life-saving actions should be accepted for a short period of time, but if lack of intervention by medical and support teams is extended, the consequences will include deterioration of the quality of life of this population. ${ }^{5,6}$

$\mathrm{WHO}^{7}$ describes the considerations on disability during confinement by COVID-19, emphasizing the risks of contagion from differentcauses that include: barriers for the implementation of basic hygiene measures, such as hand washing (e.g. impossibility for a person with a motor disability); difficulty enacting social distancing due to intellectual disability or because they are institutionalized patients; by the need to touch things to obtain information from the environment (visual or intellectual disability) or for physical support (motor disability); emphasizing 
also in the barriers to access public health information and access to medical care. ${ }^{8}$

In times of stress and uncertainty, such as those faced in this pandemic, it is important to have strategies for managing different situations that children with disabilities may face, such as abrupt changes in routines, loss of connections to friendly environments (friends, therapists), inability to accept confinement, and the probability of presenting symptoms such as anxiety or depression. ${ }^{9}$ For this reason, family and caregivers, as well as health professionals, including pediatric dentists, play an important role in helping to understand the changes and process their related feelings.

For example, in cases of children with ASD (Autism Spectrum Disorders) Hume, K. et al. $^{10}$ propose a seven item strategy to support people with autism during these uncertain times: 1. Support for understanding, 2. Offer opportunities for expression, 3. Give priority to coping and relaxation skills, 4. Maintain routines, 5. Build new routines, 6. Encourage connections (from distance), 7. Manage behavioral changes. In many cities around the world, special permits have been granted for patients with ASD or other disabilities to take accompanied walks with a person to avoid symptoms of anxiety or other. ${ }^{11}$

It is important to continue medical monitoring of children with associated systemic diseases, such as Down Syndrome $^{12}$ which in 40 to $60 \%$ have congenital cardiopathies and in severe cases respiratory compromise. ${ }^{13}$
Pediatric dental care strategy during confinement stage of the COVID-19 pandemic.

This strategy is based on the recommendations of ALOP - Latin American Association of Pediatric Dentistry - regarding the care route for Pediatric Dentistry procedures during the confinement or quarantine stage of the COVID-19 pandemic. ${ }^{14}$ Recommendations are added to this guide according to the different needs of children with disabilities and medically compromised, always bearing in mind that only emergency dental care will be performed. (Figure 1)

\section{Tele-dentistry}

Distance dental care (tele-dentistry ${ }^{15}$ ), is the first line of care in exceptional cases that may arise in public health crises and when on site contact between the patient and the specialist is not possible due to geographical distances or other restrictions. ${ }^{15,16}$

Tele-dentistry should be considered as a fundamental tool to be able to evaluate the degree of urgency/emergency for dental care. The use of this modality in pediatric dentistry in children with disabilities or medically compromised will follow the guidelines of ALOP 2020, ${ }^{15}$ emphasizing the importance of being able to resolve the emergency in this way and continue with the virtual follow-up and thus avoid the risk of transfer due to constitute a group of greater risk in case of SARS-CoV-2 infection.

The medical/dental questionnaire will be mainly addressed to the parents or careers of the children, and in those patients medically 


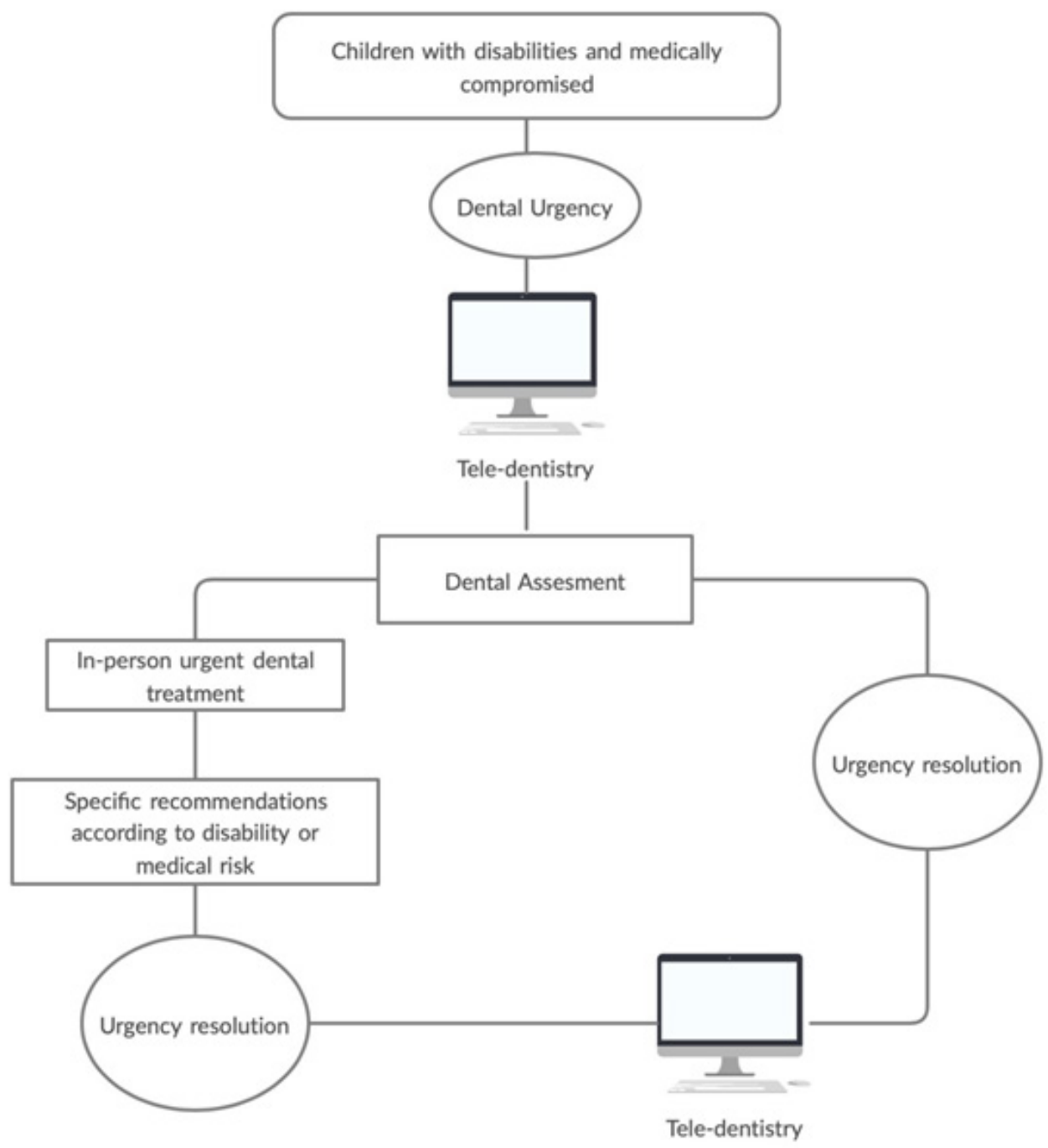

Figure 1. Intervention flowchart for the resolution of dental in patients with disabilities

compromised, inter-consultation with their corresponding health team should be carried out. It is important in these cases to request contact with the family physician in order to make the inter-consultations if necessary. Virtual triage will be performed following the "Pediatric Emergency follow -up sheet COVID-19 pandemic" (ALOP $2020^{15}$ ) and thus obtain information on the patient's symptomatology in order to provide guidance and decide on a followup route.

The application of telemedicine/teledentistry care must take into account that there are gaps in access to computers and the Internet and limitations in skills for their use in homes with people with disabilities. ${ }^{17}$ In some children it may be difficult for the pediatric dentist to recognize the specific urgency due to different factors, for example, uncooperative behavior that prevents acceptable obtaining acceptable intraoral images, or because of the inability of the family or caregivers to understand or figure out the cause of the emergency. In these cases it will be necessary to organize outpatient and on-site care for the resolution of the emergency. Once the patient's appointment has been decided, ideas are exchanged with the parents or 
caregivers in order to prepare the child for the attendance. This can be done by sending material (videos, pictograms, etc.) according to the condition of each patient in order to reinforce the motivation that will be done in person.

\section{On-site appointment}

This paper suggests different strategies to adapt children to the urgent/emergent dental situation, ${ }^{17}$ taking into account the conditions of disability or complex systemic disease that they present, using behavioral and motivational modelling strategies, and also taking into account both the protection of health personnel (dentists, assistants and administrative staff) and the protection of the child and his or her companion to prevent the transmission of SARS-CoV-2. ${ }^{19,} 20$

\section{Before admission to the dental office:}

The Pediatric dentist will be dressed with appropriate surgical gown and wearing a surgical or inclusive mask ${ }^{21}$ which allows visualization of the mouth and its movement when speaking, which is recommended, for example, for hearing impaired children.

A distance of two meters will always be kept when receiving the patient at the door of the office. Temperature will be taken and triage will be done in person with an interview that includes the child's medical condition. The dental assistant will be dressed in the personal protective equipment (PPE) ${ }^{22,23}$ to assist the dentist in dressing with the same biosecurity measures (PPE). It is recommended that the child with an intellectual disability or autism spectrum disorder (ASD) witness, with the indicated physical distance, the entire placement maneuver of the different elements to be used by the professional (Protocol indicated in the Care Route for ALOP Pediatric Dentistry procedures ${ }^{14}$ ) and thus decrease the fear and anxiety that may be caused by the clothing required for attention. It is suggested to avoid excesses in the use of clothing that only add more possibilities of mistakes when discarding it and do not really provide greater biosecurity.

It is recommended to work though the previously described procedures taking advantage of Tele-dentistry, providing different resources to parents or caregivers to achieve adaptation to the dental environment. The sequence of the procedure must be explained to the parents or caregivers in order to be able to work the steps with the child at home. Possible difficulties in communication and cognitive accessibility should be considered and implementing augmentative communication, through the use of pictograms in order to explain the origin of this disease, symptoms, hygiene habits and new routines. ${ }^{24,25}$ The same pictograms or videos will be used in on-site appointment before admission to the office to reinforce the child's motivation or adaptation to the new situation. ${ }^{26,27}$ The ARASAAC institution makes available on its website materials to offer to families, therapists and professionals (Figures 2 and 3). ${ }^{28}$

\section{At the dental office:}

1. Before starting patient care, the patient must rinse with a $1 \%$ hydrogen peroxide mouthwash or a $0.2 \%$ iodopovidone mouthwash for 1 minute (after ruling out iodine allergy). In the majority of pediatric patients younger than 4 years, 


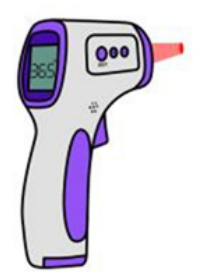

Thermometer
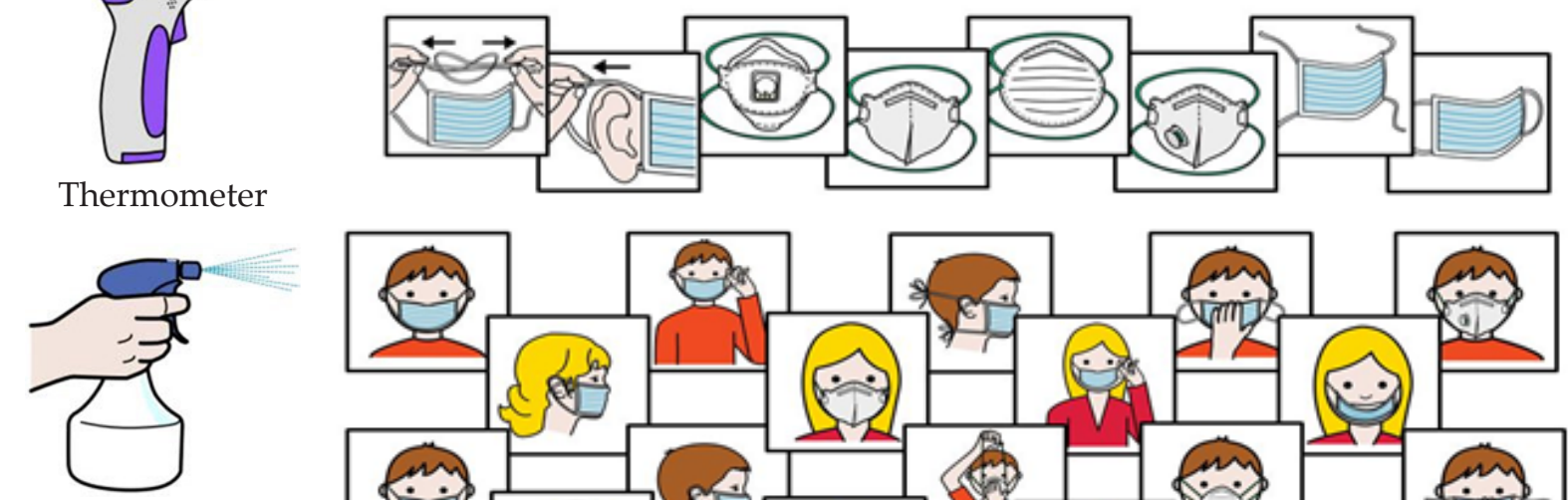

Sanitizer for surfaces and clothing
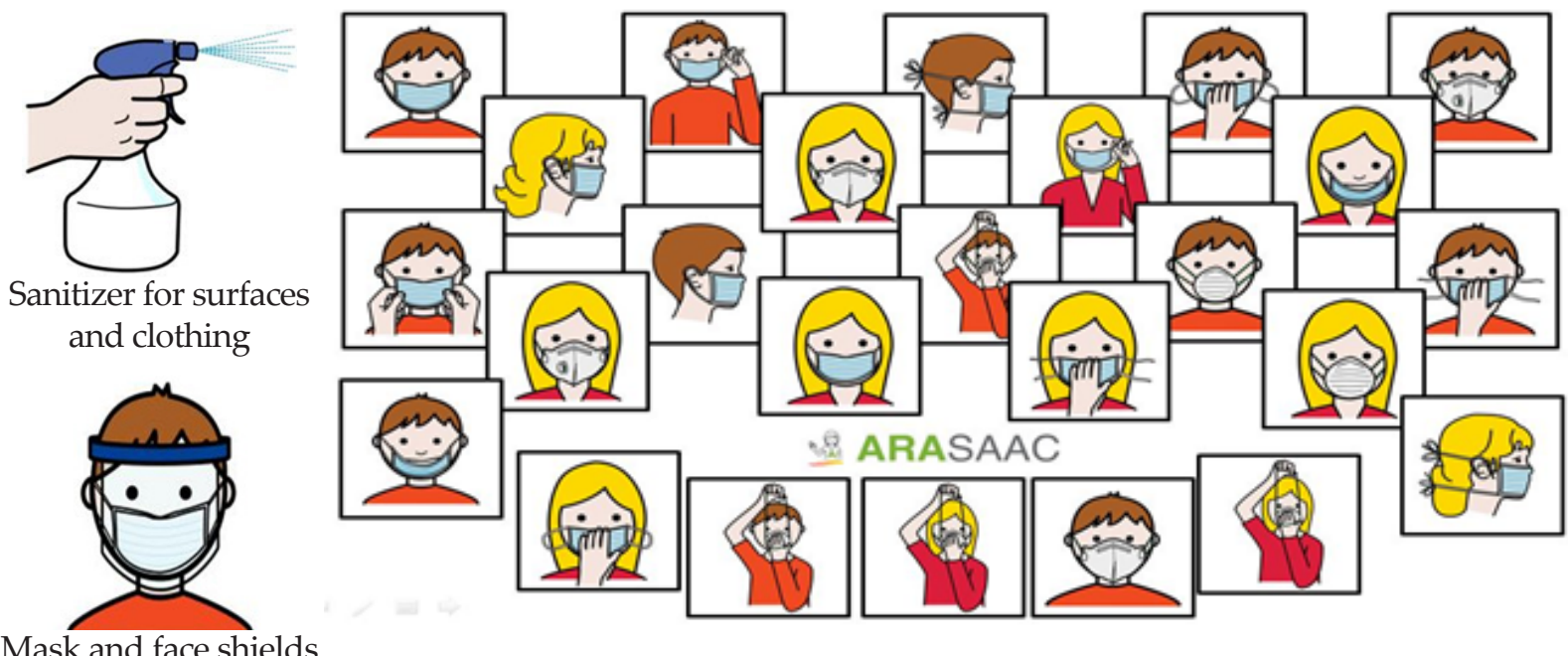

Mask and face shields

Figura 2. Pictograms of materials and masks. Source: ARASAAC http://www.arasaac.org/materiales.php?id_material $=2357$

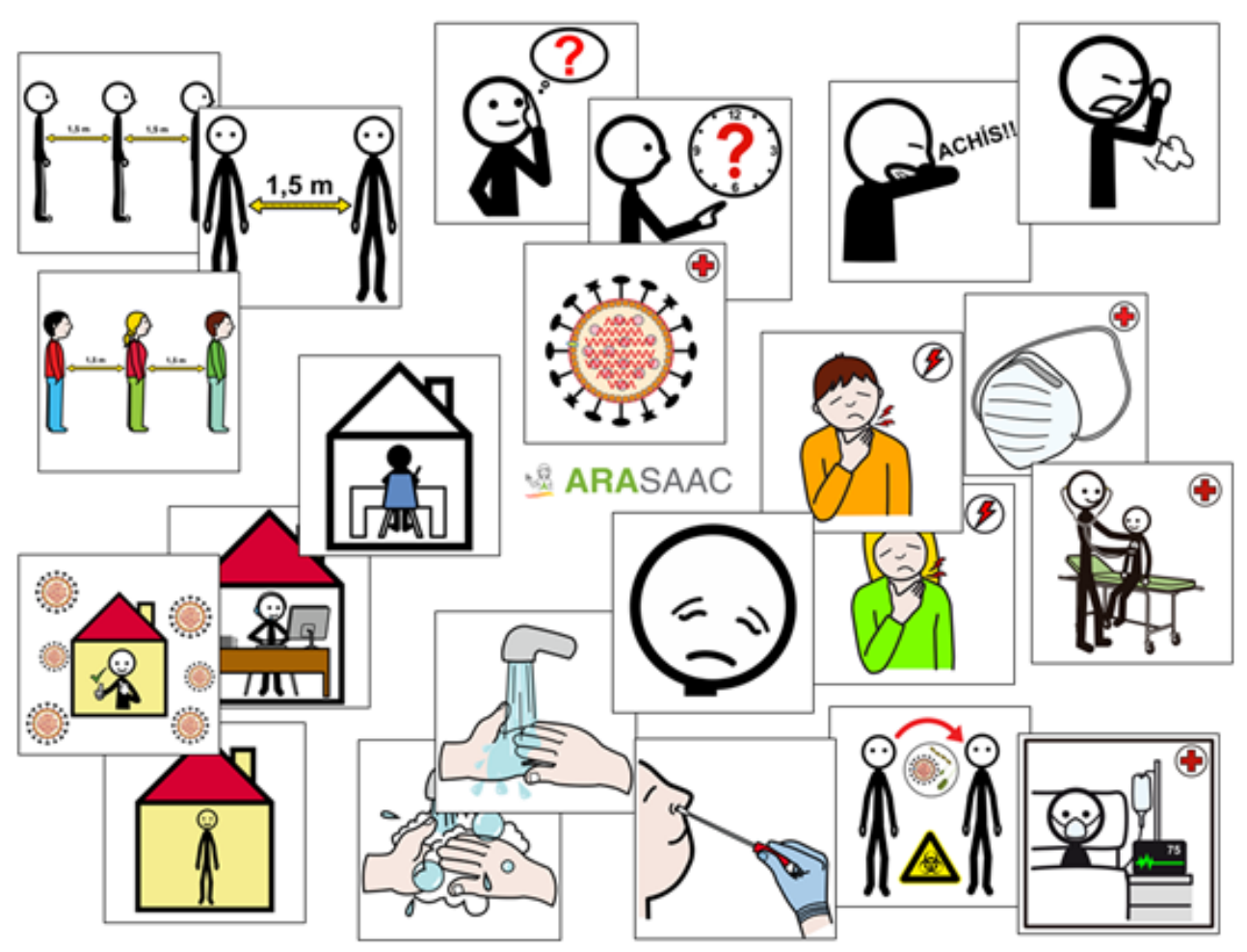

Figura 3. Pictograms of recommended behaviors during the COVID-19 pandemic. Source: ARASAAC http://www.arasaac.org/materiales.php?id_material=2357 
as well as those children with motor, intellectual, sensory disabilities or those patients with difficult behavior, rinsing is not indicated due to the inability to do so, or to reduce the risk of ingestion. For these cases, cleaning the oral cavity with gauze, cotton roll or brush impregnated with hydrogen peroxide is performed. The choice of hygiene modality can be decided with the parents or caregivers.

2. In cases of patients with CNPE (Chronic non-progressive encephalopathy) or with other motor disorders, it is suggested, prior to the intraoral procedure, to perform orofacial massages in order to decrease spasticity and relax the child appropriately. ${ }^{29}$

3. The use of high speed handpieces should be minimized, prioritizing nonaerosol generating procedures. ${ }^{30,} 31$ It should be considered that children with ASD or sensory disorders are very sensitive to noise and the minimally invasive caries approach is preferred, for example with techniques such as a traumatic (ART) or the application of silver diamine. ${ }^{30,32}$

4. The use of the rubber dam for operative or endodontic procedures, is indicated whenever possible. In some cases cyanoacrylate, elastic bands, and dental floss can be used to replace the use of clamps or staples. ${ }^{33,34}$

5. The use high suction is recommended, if possible. Noise can trigger unwanted behavior as well as the use of rotating instruments in children with sensory disorders and high sensitivity so it is recommended to use low suction or headphones in addition to music to decrease the perception. ${ }^{35}$

6. Working with four-handed dentistry is essential. ${ }^{31} \mathrm{~A}$ trained dental assistant is a fundamental pillar in both dental care and help during the motivation of the child with a disability and the support of their parents or caregivers.

7. During the treatment, only the patient, operator and assistant should be in the room. If necessary, allow a maximum of one companion per patient. In patients who allow it, the office door can be kept open and the companion can be on the threshold of the door so that the child can observe him and even participate in the dental situation, calming the patient.

8. The patient must be able to collaborate with the treatment being performed. To do this, use proper psychological strategies for behavioral management. In cases of patients who are unable to collaborate with treatment, refer for care under sedation or general anesthesia. ${ }^{31,36,37}$ In cases of referral to that modality of care, the child must not present acute respiratory pathology (symptomatic COVID-19), and the dental urgency will be controlled with symptomatic measures (control of infection and pain with antimicrobials and analgesics, if applicable).

Concerns have been expressed regarding the risk of aerosol generation in the administration of nitrous oxide analgesia but so far, there are no studies directly related to this potential problem. ${ }^{36} \mathrm{Clinical}$ guidelines advise the use of nitrous oxide and other forms of conscious sedation if urgent care is required in patients who do not allow conventional treatment. It is also recommended to avoid the use of general anesthesia only for patients with poor oral health that can impact and aggravate pre-existing medical 
conditions as well as children with selfinjurious behavior due to pain. ${ }^{31,38}$

\section{Conclusion}

Pediatric dentistry must ensure accessibility to dental care for children with disabilities or those medically compromised, knowing the emotional, psychological and physical impact that this COVID 19 pandemic is causing in the general population and these children in particular.

For this reason, it is necessary for the pediatric dentist to anticipate, together with the families and the interdisciplinary team, the new routines to facilitate care by taking measures such as tele-dentistry prior to the consultation, preparing children to accept the dental environment with the appropriate biosecurity measures, as well as promoting the use of alternative communication alluding to the current pandemic and in this way contributing to the desensitization of the child when facing dental care.

\section{Autors}

Development of this article led by Gabriela Scagnet (Argentina), Jorgelina Valente (Argentina), Ana Clara Zabala (Argentina), Mariana Armada (Argentina), Laura Fedelli (Argentina), Lina María Hernández Salas (Colombia), on behalf of the COVID-19 Interdisciplinary team, Latin-American Pediatric Dentistry Association (Asociación Latinoamericana de Odontopediatría).
COVID-19 Interdisciplinary team, Latin-American Pediatric Dentistry Association (Asociación Latinoamericana de Odontopediatría):

Jenny Abanto (Brasil), Mariana Armada (Argentina), Paola Beltri (España), Marisol Carrillo Tabakman (Paraguay), Haydée Casaretto (Argentina), Jorge Luis Castillo (Perú), Mónica Gladys Cesetti (Argentina), Bertha Angélica Chávez González (Perú), Ana Claudia Rodrigues Chibinski (Brasil), Salomon Alberto Cohen (Argentina), Olga Cortés Lillo (España), Luzia Ana Da Silva de Carballo (Venezuela), Gonzalo De la Fuente Alvarez (Chile), Renée Di Nallo (Argentina), María Débora Elizabeth Dricas (Argentina), Sandra Echevarria (Brasil), Piedad Cecilia Echeverry Marin (Colombia), Laura Fedelli (Argentina), Carlos Flores-MIr (Canadá), Andrea Virgina González Carfora (Chile), Lina María Hernández Salas (Colombia), Francisco José Hernández Restrepo (Colombia), Maria Teresa Ibañez Rodriguez (Bolivia), José Carlos Pettorossi Imparato (Brasil), Alejandra Lipari Valdés (Chile), Daniela Madrigal López (Costa Rica), Daniela Catalina Martínez Camus (Chile), María Gabriela Martínez Vásquez (Venezuela), A. Carolina Medina Díaz (Venezuela), Kelly Maria Silva Moreira (Brasil), Elías M. Morón (Estados Unidos de América), Martha Mutis (Estados Unidos de América), Camila Palma (Perú), Gladys Mabel Peña (Argentina), Adriana Pistochini (Argentina), Paloma Planells (España), Gabriel Politano (Brasil), Matias RiosErazo (Chile), Adriana Maria Rubiano Pinzon (Colombia), Karla Mayra Rezende (Brasil), Rosa Gabriela Rondón (Venezuela), Gabriela Scagnet (Argentina), Rosemary Sogbe de Agell (España), Marina Tavares Costa Nóbrega (Canadá), Jorgelina Valente (Argentina), Ernesto Venegas De Herrera (República Dominicana), Rosa Helena Wanderley Lacerda (Brasil), Ana Clara Zabala (Argentina), Gabriel Zambrano (Venezuela).

Información completa del Grupo Interdisciplinario COVID-19 de la Asociación Latinoamericana de Odontopediatría

\section{References}

1. United Nations International Children's Emergency Fund. UNICEF. Respuesta COVID-19: consideraciones para niñas, niños y adultos con discapacidades.2020. Disponible en :https://www. unicef.org/disabilities/files/RESPUESTA_COVID_-_SPANISH.pdf

2. Morales-Chávez M. Coronavirus y discapacidad - Una población muy vulnerable. Acta Odontol. Venez. Edición Especial: COVID-19. 2020.

3. Campbell VA, Gilyard JA, Sinclair L, Sternberg T, Kailes JI. Preparing for and responding to pandemic influenza: Implications for people with disabilities. Am. J. Public Health. 2009; 99(S2): S294-S300. Disponible en: https://ajph.aphapublications.org/doi/10.2105/AJPH.2009.162677 
4. Negrini S, Grabljevec K, Boldrini P, Kiekens C, Moslavac S, Zampolini M, et al. Up to 2.2 million people experiencing disability suffer collateral damage each day of Covid-19 lockdown in Europe. Eur J Phys Rehabil Med. 2020; May 08. DOI: 10.23736/S1973-9087.20.06361-3. Disponible en: https://doi. org/10.23736/S1973-9087.20.06361-3

5. European Physical and Rehabilitation Medicine Bodies Alliance, Negrini S. White Book on Physical and Rehabilitation Medicine in Europe. Introductions, Executive Summary, and Methodology. European J Phys Rehabil Med. 2018; 54(2): 125-155. Disponible en: https://doi.org/10.23736/S1973-9087.18.05143-2.

6. World Health Organization.WHO. Critical preparedness, readiness and response actions for COVID-19. Disponible en: https://www.who.int/publications-detail/critical-preparedness-readinessand-response-actions-for-covid-19

7. World Health Organization. WHO: Disability considerations during the COVID-19 outbreak Disponible en: : https://www.paho.org/en/documents/disability-considerations-during-covid-19-outbreak

8. Noticias Organización Naciones Unidas. ONU. Las personas con discapacidad, en riesgo por el coronavirus. [Internet] [Consultado 19 Marzo 2020]. Disponible en: https://news.un.org/es/ story/2020/03/1471282

9. Hume K, Waters V, Sam A, Steinbrenner J, Perkins Y, Dees B., et al. Supporting individuals with autism through uncertain times. Chapel Hill, NC: School of Education and Frank Porter Graham Child Development Institute, University of North Carolina at Chapel Hill. 2020. Disponible en :A https:// afirm.fpg.unc.edu/supporting-individuals-autism-through-uncertain-times

10. Lima CKT, Carvalho PMM, Lima IAAS, Nunes JVAO, Saraiva JS, de Souza RI, et al. The emotional impact of Coronavirus 2019-nCoV (new Coronavirus disease). Psychiatry Res. 2020; 12:112915.

11. Autism Spaeks.USA: Autism Speak; what should the autism community know about the coronavirus outbreak?; c2020; [cited 2020 March 4] Disponible en : https://www.autismspeaks.org/science-news/ what-should-autism-community-know-about-coronavirus-outbreak

12. Scagnet G. Actualización odontológica en la atención del niño con síndrome de Down. Odontol. pediatr. 2013; 12(1): 27-40. Spanish. Disponible en: http://repebis.upch.edu.pe/articulos/op/v12n1/a4.pdf

13. Medrano CL., García-Guereta LS, Lirio JC, García JP. Respiratory infections, Down's syndrome and congenital heart disease: the CIVIC 21 study. An Pediatr. 2009; 71(1): 38-46. Español. Disponible en : https://doi.org/10.1016/j.anpedi.2008.11.007

14. Asociación Latinoamericana de Odontopediatría. Grupo COVID-19.Ruta de atención para procedimientos de Odontología Pediátrica durante la etapa de confinamiento o cuarentena de la pandemia COVID-19. 2020; 10 (2).Disponible en: https://www.revistaodontopediatria.org

15. Asociación Latinoamericana de Odontopediatría. Grupo COVID-19. Tele dentistry Guidelines in Pediatric Dentistry during the COVID-19 pandemic. 2020; 10, (2).Disponible en: https://www. revistaodontopediatria.org/ediciones/2020/2/art-3

16. Jampani ND, Nutalapati R, Dontula BS, Boyapati R. Applications of teledentistry: A literature review and update. J Int Soc Prev Community Dent. 2011; 1(2):37-44. doi:10.4103/2231-0762.97695.

17. Economic Commission for Latin America and the Caribbean. ECLAC. Persons with disabilities and coronavirus disease (COVID-19) in Latin America and the Caribbean: status and guidelines.2020. Disponible en: https://www.cepal.org/en/publications/45492-persons-disabilities-and-coronavirusdisease-covid-19-latin-america-and-caribbean

18. Polli V, Sordi M, Lisboa M, Munhoz E, Camargo A. Dental Management of Special Needs Patients: A Literature Review . Glob J Oral Sci.2016 ; (2):33-45.

19. Centers for Disease Control and Prevention. CDC.Coronavirus Disease 2019; Interim infection prevention and control recommendations for patients with suspected or confirmed coronavirus disease 2019 (COVID-19) in healthcare settings. 2020; March 19. Disponible en: https://www.simlaweb. it/wp-content/uploads/2020/03/Infection-Control-Severe-acute-respiratory-syndrome-coronavirus-2SARS-CoV-2-CDC.pdf

20. American Academy of Pediatric Dentistry. COVID-19 Update/Coronavirus Update. 2020; April. Disponible en : https://www.aapd.org/about/about-aapd/news-room/covid-19/.

21. Federación de Asociaciones de Personas con Discapacidad Física y Orgánica de Córdoba. COCEMFE. Mascarillas solidarias. 2020; April. Disponible en: https://cordobainclusiva.com/mascarilla-solidaria 
22. Centers for Disease Control and Prevention CDC. Recommendation: Postpone Non-Urgent Dental Procedures, Surgeries, and Visits. 2020; March 27. Disponible en: https://www.cdc.gov/oralhealth/ infectioncontrol/statement-COVID.html.

23. Verbeek JH, Rajamaki B, Ijaz S, Sauni R, Toomey E, Blackwood B, Tikka C, Ruotsalainen JH, Kilinc Balci FS.; Cochrane Collaboration; Cochrane Database of Systematic Reviews. Personal protective equipment for preventing highly infectious diseases due to exposure to contaminated body fluids in healthcare staff. 2020(5) CD011621. Disponible en: https://www.cochranelibrary.com/es/cdsr/ doi/10.1002/14651858.CD011621.pub3/full/es

24. Confederación Autismo España. Gobierno de Aragón: Centro Aragonés para la Comunicación Aumentativa y Alternativa. ARASAAC. 2020. Disponible en : http://www.autismo.org.es/actualidad/ articulo/como-explicar-el-covid-19-las-personas-con-tea:\%20ARASAAC (www.arasaac.org)

25. Gobierno de Aragón: Centro Aragonés para la Comunicación Aumentativa y Alternativa. ARASAAC. Pictogramas de conductas recomendadas durante la pandemia COVID-19. (http:// arasaac.org Licencia: CC (BY-NC-SA) Disponible en: https://drive.google.com/file/d/1uLDQMF_ rGn3VPxkWmqGJYFVv89EWkuOD/view

26. Aspanaes. Material accesible para personas con TEA para comprender el Coronavirus. Pictograma. 2020. Disponible en: https://drive.google.com/file/d/1MC1HuPq3KHywm4z7rKDgB-rcjYnzUOdE/ view.

27. Molina M. COVIBOOK .2020. Available from: https://660919d3-b85b-43c3-a3ad-3de6a9d37099. filesusr.com/ugd/64c685_0a595408de2e4bfcbf1539dcf6ba4b89.pdf open?id=10xY03EV8mvK78m09NR5Pm2qB1uJ0xDgq:

28. Centro Aragonés para la Comunicación Aumentativa y Alternativa ARASAAC. Disponible en: http:// aulaabierta.arasaac.org/materiales-caa-coronavirus)

29. García, M. D. E., \& Zambrano, V. P. P. Parálisis cerebral infantil: estimulación temprana del lenguaje método de Bobath. Dominio de las Ciencias. 2017. Jun 20; 3(4), 627-706.

30. AsociaciónLatinoamericanadeOdontopediatría:TratamientodecariesenépocadeCOVID-19:Protocolos clínicos para el control de generación de aerosoles. Revista de Odontopediatría Latinoamericana. 2020; (10) 2. Disponible en: https://www.revistaodontopediatria.org/ediciones/2020/2/art-2/

31. International Association for Disability \& Oral Health. IADH. Second Edition of the IADH Fact Sheet COVID-19. 2020; April 15. Disponible en: http://iadh.org/news/updated-iadh-covid-fact-sheet-2-1/

32. Mallineni SK, Innes NP, Raggio DP, Araujo MP, Robertson MD, Jayaraman J. Coronavirus disease (COVID-19): Characteristics in children and considerations for dentists providing their care. Int . Paediatr Dent. 2020; 30(3), 245-250.

33. Torres Diaz, E. (2017). Aislamiento del campo operatorio en odontopediatría http://repositorio.uigv. edu.pe/handle/20.500.11818/1572.

34. González González JM. Cianoacrilato: Definición y propiedades. Toxicidad y efectos secundarios. Aplicaciones en medicina y odontología. Av Odontoestomatol. 2012; 28(2): 95-102.

35. Guzman G, Putrino N, Martinez F, Quiroz N. Nuevas tecnologías: Puentes de comunicación en el trastorno del espectro autista (TEA). Ter Psicol.2017; 35(3): 247-258. Disponible en: https://scielo. conicyt.cl/pdf/terpsicol/v35n3/0716-6184-terpsicol-35-03-0247.pdf . Spanish

36. Souza, R. C. C., Costa, P. S., \& Costa, L. R. Precauções e recomendações sobre sedação odontológica durante a pandemia de COVID-19. Rev Bras Odontol. April 2020; 77. Portuguese

37. Giordano CE, Giordano CL, Cunha-Correia AS. Sedação inalatória com óxido nitroso para assistência odontológica durante a pandemia de COVID-19. Revista Faipe. 2020; 10(1): 69-84. Disponible en: http://revistafaipe.lifesistemas.com.br/index.php/RFAIPE/article/view/196/141 Portuguese

38. Royal College of Surgeons of England, Recommendations for Special Care Dentistry during Covid-19 pandemic. April 2020. Disponible en : www.rcseng.ac.uk/dental-faculties/fds/coronavirus/

Recibido/Received: 01/06/2020

Modificaciones aceptadas/Final version: 07/06/2020

Correspondencia: e-mail: editor@revistaodontopediatria.org 